\title{
Mapping spaces in quasi-categories
}

\author{
DANIEL DUGGER \\ DAVID I SPIVAK
}

\begin{abstract}
We apply the Dwyer-Kan theory of homotopy function complexes in model categories to the study of mapping spaces in quasi-categories. Using this, together with our work on rigidification from [8], we give a streamlined proof of the Quillen equivalence between quasi-categories and simplicial categories. Some useful material about relative mapping spaces in quasi-categories is developed along the way.
\end{abstract}

55U40; 18G30, 18B99

\section{Introduction}

A quasi-category is a simplicial set $X$ with a certain extension property, weaker than the classical Kan condition. This property ensures that the simplicial set behaves like a higher-dimensional category; that is, $X$ can be thought of as something like a category with $n$-morphisms for any $n \geq 1$. More precisely, one nowadays says that quasi-categories give a model for " $(\infty, 1)$-categories", because the $n$-morphisms for $n>1$ are all invertible. Quasi-categories were introduced briefly by Boardman and Vogt [3], and have since been studied by Cordier and Porter [5], Joyal [13; 15] and Lurie [16], among others.

If $K$ is a quasi-category and $x$ and $y$ are $0-$ simplices, it is possible to construct a mapping space $K(x, y)$ which is a simplicial set. The trouble is that there are many different ways to exhibit such mapping spaces, the different models being weakly homotopy equivalent but not isomorphic. No particular model is ideal for every application, and so one must become versatile at changing back-and-forth. In [16] Lurie uses several models, most prominently the ones denoted there $\operatorname{Hom}_{K}^{R}(x, y)$, $\operatorname{Hom}_{K}^{L}(x, y)$, and $\mathfrak{C}(K)(x, y)$. In order to understand the connections between these, Lurie develops a theory of "straightening and unstraightening functors," which is reasonably complicated.

This paper has two main goals. First, we explain how the mapping spaces of quasicategories fit into the well-understood theory of homotopy function complexes in model categories; see Dwyer and Kan [10]. The latter technology immediately gives various 
tools for understanding why different models for these mapping spaces are weakly equivalent. Lurie's $\operatorname{Hom}_{K}^{R}(x, y)$ and $\operatorname{Hom}_{K}^{L}(x, y)$-as well as two other useful models (see Proposition 4.5)—fit directly into this framework, but $\mathfrak{C}(K)(x, y)$ does not. Our second goal is to use these tools, together with our work on $\mathfrak{C}(-)$ from [8], to give a direct proof that the $\mathfrak{C}(K)$ mapping spaces are weakly equivalent to the Dwyer-Kan models. This in turn allows us to prove that the homotopy theory of quasi-categories is Quillen equivalent to that of simplicial categories. This result was originally proved by Joyal [14] (unpublished) and Lurie [16], but we give a new proof here. Our proof avoids the use of Lurie's straightening and unstraightening functors.

We now describe the contents of the paper in more detail. Recall that a quasi-category is a simplicial set $X$ that satisfies the extension condition for the so-called inner horn inclusions-the inclusions $\Lambda_{k}^{n} \hookrightarrow \Delta^{n}$ for $0<k<n$. It turns out that there is a model category structure on $s$ et where the cofibrations are the monomorphisms, the fibrant objects are the quasi-categories, and where the weak equivalences are something we will call Joyal equivalences. See Section 2 for more background. We will call this the Joyal model structure, and denote it $s \mathscr{S}_{\boldsymbol{e}}{ }_{J}$. The classical weak equivalences of simplicial sets-maps that induce homotopy equivalences of the geometric realizations-will be termed Kan equivalences from now on.

In any model category $\mathcal{M}$, given two objects $X$ and $Y$ there is a homotopy function complex $\operatorname{hMap}_{\mathcal{M}}(X, Y)$. The theory of these objects is largely due to Dwyer and Kan $[11 ; 9 ; 10]$. Such a function complex can be defined in several ways, all of which are Kan equivalent:

(1) It is the mapping space between $X$ and $Y$ in the simplicial localization $L_{W} M$, where one inverts the subcategory $W$ of weak equivalences; see [11].

(2) It is the mapping space in the hammock localization $L^{H} \mathcal{M}$ constructed by Dwyer and Kan in [9].

(3) It can be obtained as the simplicial set $[n] \mapsto \mu\left(Q^{n} X, \hat{Y}\right)$ where $Y \rightarrow \hat{Y}$ is a fibrant replacement in $\mathcal{M}$ and $Q^{\bullet} X \rightarrow X$ is a cosimplicial resolution of $X$ [10].

(4) It can dually be obtained as the simplicial set $[n] \mapsto \mu\left(\tilde{X}, R_{n} Y\right)$ where $\tilde{X} \rightarrow X$ is a cofibrant replacement and $Y \rightarrow R_{\bullet} Y$ is a simplicial resolution of $Y$.

(5) It can be obtained as the nerve of various categories of zigzags, for instance (if $Y$ is fibrant) the category whose objects are zigzags

$$
X \stackrel{\sim}{\longleftarrow} A \longrightarrow Y
$$

and where the maps are commutative diagrams equal to the identity on $X$ and $Y$. 
In some sense the models in (3) and (4) are the most computable, but one finds that all the models are useful in various situations. One learns, as a part of model category theory, how to compare these different models and to see that they are Kan equivalent. See [10] and the first author's paper [6], as well as Section 3 of the present paper.

The above technology can be applied to the Joyal model structure in the following way. The overcategory $\left(\partial \Delta^{1} \downarrow s \mathscr{S e t}_{J}\right)$ inherits a model category structure from $s \mathscr{S e t}_{J}$ (see Hirschhorn [12, 7.6.5]). Given a simplicial set $K$ with chosen vertices $a$ and $b$, consider $K$ as a simplicial set under $\partial \Delta^{1}$ via the evident map $\partial \Delta^{1} \rightarrow K$ sending $0 \mapsto a, 1 \mapsto b$. In particular, we can apply this to $\Delta^{1}$ and the vertices 0 and 1 . This allows us to consider the homotopy function complex

$$
\operatorname{hMap}_{\left(\partial \Delta^{1} \downarrow_{s} \varphi_{\left.e t_{J}\right)}\right.}\left(\Delta^{1}, K\right),
$$

which somehow feels like the pedagogically "correct" interpretation of the mapping space in $K$ from $a$ to $b$-it is a purely homotopical description, not depending on any particular choice of model, and it generalizes a similar description of morphisms in a 1 -category as maps from the classical arrow category [1].

Once we have the appropriate definitions in place, the following result becomes an easy observation (it is restated and proved as Corollary 4.8):

1.2 Proposition Let $K$ be a quasi-category. The mapping spaces $\operatorname{Hom}_{K}^{R}(a, b)$ and $\operatorname{Hom}_{K}^{L}(a, b)$ of Lurie [16] are models for the homotopy function complex (1.1), obtained via two different cosimplicial resolutions of $\Delta^{1}$. The pullback

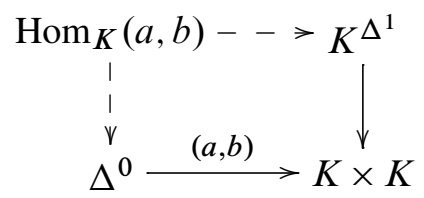

is also a model for the same homotopy function complex, this time obtained via a third cosimplicial resolution, namely the one sending $[n]$ to the pushout

$$
\Delta^{1} \times \Delta^{n} \leftarrow \partial \Delta^{1} \times \Delta^{n} \rightarrow \partial \Delta^{1} .
$$

Note that, given the above proposition, the Dwyer-Kan technology shows immediately that the three constructions $\operatorname{Hom}_{K}^{R}(a, b), \operatorname{Hom}_{K}^{L}(a, b)$ and $\operatorname{Hom}_{K}(a, b)$ are all Kan equivalent, and in fact gives a "homotopically canonical" weak equivalence between any two. 


\subsection{Connections with the $\mathfrak{C}$ mapping spaces}

One problem with using the Dwyer-Kan models in our setting is that given 0-simplices $a, b$ and $c$ in a quasi-category $K$, there do not exist naturally arising composition maps

$$
\operatorname{hMap}\left(\Delta^{1}, K_{b, c}\right) \times \operatorname{hMap}\left(\Delta^{1}, K_{a, b}\right) \longrightarrow \operatorname{hMap}\left(\Delta^{1}, K_{a, c}\right) .
$$

(Here the mapping space is taken in the category ( $\partial \Delta^{1} \downarrow s \mathscr{S e t}_{J}$ ), as in (1.1), and the notation $K_{x, y}$ tells us to regard $K$ as an object in this category via the map $\partial \Delta^{1} \rightarrow K$ sending $0 \rightarrow x$ and $1 \rightarrow y$ ). In [16] Lurie gives a functor $\mathfrak{C}: s \mathscr{S e t} \rightarrow s \mathscr{C a t}$, and the simplicial sets $\mathfrak{C}(K)(a, b)$ can be thought of as models for function complexes of $K$ which do admit such composition maps. In [8] we gave another functor $\mathfrak{C}^{\text {nec }}$ which accomplished the same task, and we proved that the mapping spaces $\mathfrak{C}(K)(a, b)$ and $\mathfrak{C}^{\text {nec }}(K)(a, b)$ were naturally Kan equivalent. In fact we gave an entire collection of different models $\mathfrak{C}^{\mathcal{G}}$, all of which were Kan equivalent. What is not immediately clear is how to connect the Dwyer-Kan function complexes to the mapping spaces arising in $\mathfrak{C}, \mathfrak{C}^{\text {nec }}$ or $\mathfrak{C}^{\mathfrak{G}}$. This is explained in Section 5, where they are shown to be connected by a canonical zigzag of Kan equivalences.

At one level the connection can be seen as follows. Recall from [8] that a necklace is a simplicial set of the form $\Delta^{n_{1}} \vee \cdots \vee \Delta^{n_{k}}$, obtained from simplices by gluing the final vertex of one to the initial vertex of its successor. There is a natural linear order on the vertices of a necklace, and there is a unique 1-simplex connecting each nonterminal vertex to its successor. The spine $\operatorname{Spi}[T]$ of a necklace $T$ is the union of these 1-simplices, and the associated simplex $\Delta[T]$ of $T$ is the simplex with the same ordered vertex set as $T$.

The mapping space $\mathfrak{C}^{\text {nec }}(K)(a, b)$ is just the nerve of the evident category whose objects are pairs $\left[T, T \rightarrow K_{a, b}\right]$ where $T$ is a necklace and $T \rightarrow K$ is a map sending the initial and final vertices of $T$ to $a$ and $b$. The mapping spaces $\mathfrak{C}^{\mathcal{G}}(a, b)$ are defined similarly, but where one replaces the category of necklaces with a category $\mathcal{G}$ of some other suitable "gadgets."

For any necklace $T$, there is a canonical inclusion $T \hookrightarrow \Delta[T]$; it may be checked that this inclusion is a weak equivalence in $s \mathscr{S e t}_{J}$ (see Lemma 9.1). Any map $T \rightarrow K$ therefore gives rise to a zigzag

$$
\Delta^{1} \longrightarrow \Delta[T] \stackrel{\sim}{\longleftarrow} T \rightarrow K
$$

where the map $\Delta^{1} \rightarrow \Delta[T]$ is the unique $1-$ simplex connecting the initial and final vertices of the simplex $\Delta[T]$. Zigzags of the above type are known to give a model for homotopy function complexes (see Section 3). 
The considerations from the above paragraphs lead to a comparison map between the $\mathfrak{C}^{\text {nec }}(K)(a, b)$ mapping spaces and the Dwyer-Kan mapping spaces. This map turns out to be a Kan equivalence, although it should be noted that our proof of this is not direct: it is a crafty argument using one of the $\mathfrak{C}^{\mathcal{G}}$ constructions where one replaces necklaces by a more general class of gadgets. See Section 5 for the details. In any case, we obtain the following (where one may choose any standard model for the homotopy function complex):

1.4 Theorem Given a quasi-category $K$ with objects $a$ and $b$, there is a natural zigzag of Kan equivalences between the simplicial sets $\mathfrak{C}(K)(a, b)$ and $\operatorname{hMap}\left(\Delta^{1}, K_{a, b}\right)$.

The previous theorem is a key step in the proof of the following important result:

1.5 Theorem The adjoint pair $\mathfrak{C}:{ } \mathscr{P e t}_{J} \rightleftarrows s \mathscr{C}$ at $: N$ is a Quillen equivalence between the Joyal model structure on sYet and the model structure on $s^{6} b$ at due to Bergner [2].

We prove the above theorem as Corollary 8.2.

\subsection{Relation with the work of Lurie}

Both Theorems 1.4 and 1.5 are originally due to Lurie, and are proven in [16, 4.2.1.8 and 2.2.5.1]. In Lurie's book he starts by developing the properties of mapping spaces and rigidification and then proves the existence of the Joyal model structure as a consequence of this work. His methods involve a detailed and lengthy study of what he calls "straightening and unstraightening" functors, and it was a vague dissatisfaction with this material-together with the hope of avoiding it - that first led us to the work in the present paper.

In the present paper we start with the Joyal model structure. In both [16] and [15] it takes over a hundred pages to prove its existence, but by boiling things down to the bare essentials (and following the method of Joyal) we are able to give a streamlined approach and create the structure fairly quickly. At that point we immediately have the Dwyer-Kan techniques at our disposal, and it is through the use of these that we develop the properties of mapping spaces and rigidification. Thus, in some ways our approach is opposite that of [16].

Due to the inherent differences in the two approaches, it is slightly awkward for us to quote results from [16] without creating confusions and possible circularities. Because of this, there are a few minor results whose proofs we end up repeating or redoing in a slightly different way. The result is that the present paper can be read independently of [16] - although this should not be taken as a denial of the intellectual debt we owe to that work. 


\subsection{Organization of the paper}

The material on quasi-categories needed in the paper is all reviewed in Section 2. This material is mostly due to Joyal [15], and can also be found in Lurie [16]. Despite this, we have included three appendices in the paper where we prove all the assertions from Section 2. The reason for this is that although the results we need are reasonably straightforward, in both [16] and [15] they are intertwined with so many other things that it is difficult for a reader to extract their proofs. Especially in the case of the Joyal model structure, a basic tool in our entire approach, we felt that it was important to have a relatively short and self-contained argument. The nineteen pages in our appendices are a bit longer than ideal, but quite a bit shorter than the currently available alternatives.

Let us outline the rest of the paper. In Section 3 we review homotopy function complexes in general model categories $\mathcal{M}$. We apply these ideas in Section 4 to the case $\mathcal{M}=\left(\partial \Delta^{1} \downarrow s \mathscr{S e t}_{J}\right)$. That is, the function complex of maps $\Delta^{1} \rightarrow X$ in $\mathcal{M}$ gives the "internal mapping space" between vertices $a$ and $b$ in a quasi-category $X$. In Section 5 we compare these internal mapping spaces to the rigidification mapping spaces $\mathfrak{C}(X)(a, b)$, and in so doing rely heavily on [8]. In Section 6 we define relative mapping spaces, a useful tool for subsequent sections. In Section 7 we introduce the notion of DK-equivalence in $s \mathscr{S} t$, which provides a connection between $\mathfrak{C}$-equivalence and Joyal equivalence. In Section 8 we prove that these three notions of equivalence agree. Section 8 also provides a new proof of the Joyal/Lurie result that there is a Quillen equivalence between $s \mathscr{S e t}_{J}$ and $s_{\text {Cat }}$ (see Corollary 8.2). Finally, in Section 9 we prove some technical results that are stated without proof in Section 1 and Section 4.

\subsection{Notation and terminology}

We will use $s \mathscr{S e t}_{K}$ to refer to the usual model structure on simplicial sets, which we'll term the Kan model structure. The fibrations are the Kan fibrations, the weak equivalences (called Kan equivalences from now on) are the maps which induce homotopy equivalences on geometric realizations, and the cofibrations are the monomorphisms.

Quasi-categories are simplicial sets, and the mapping spaces between their vertices are also simplicial sets. Quasi-categories will always be viewed within the Joyal model structure, and their mapping spaces will always be viewed within the Kan model structure.

We will often be working with the category $s \mathscr{S e t}_{*, *}=\left(\partial \Delta^{1} \downarrow s \mathscr{S} t\right)$. When we consider it as a model category, the model structure we use will always be the one imported from the Joyal model structure on $s \mathscr{S e t}_{\text {; }}$ we will denote it $\left(s \mathscr{S e t}_{*, *}\right)_{J}=\left(\partial \Delta^{1} \downarrow s \mathscr{S e t}_{J}\right)$, or 
just $s \mathscr{S e t}_{*, *}$. When we write $\Delta^{1}$ as an object of $s \mathscr{S e t}_{*, *}$, we always mean to use the canonical inclusion $\partial \Delta^{1} \rightarrow \Delta^{1}$.

An object of $s \mathscr{S e t}_{*, *}$ is a simplicial set $X$ with two distinguished points $a$ and $b$. We sometimes (but not always) write $X_{a, b}$ for $X$, to remind ourselves that things are taking place in $s \mathscr{S e t}_{*, *}$ instead of $s \mathscr{S e t}$.

When $\mathscr{C}$ is a category we write $\mathscr{C}(a, b)$ instead of $\operatorname{Hom}_{\mathscr{C}}(a, b)$. We reserve the $\operatorname{Hom}_{K}(a, b)$ notation for when $K$ is a quasi-category, to denote various models of the homotopy function complex. See Proposition 1.2 or Section 4.3.

Finally, in certain places we have to deal with simplices and horns. The horn $\Lambda_{k}^{n}$ is the union of all faces containing the vertex $k$. If $\left\{m_{0}, \ldots, m_{k}\right\}$ is an ordered set, we use $\Delta^{\left\{m_{0}, \ldots, m_{k}\right\}}$ as notation for the $k$-simplex whose vertices are $m_{0}, \ldots, m_{k}$. Likewise, the " $m_{i}$-face" of $\Delta\left\{m_{0}, \ldots, m_{k}\right\}$ is the codimension one face not containing the vertex $m_{i}$, and $\Lambda_{m_{i}}^{\left\{m_{0}, \ldots, m_{k}\right\}}$ denotes the horn consisting of all faces that contain $m_{i}$. If $j_{0} \leq \cdots \leq j_{n}$, we may use "bracket notation" and write $\left[m_{j_{0}}, \ldots, m_{j_{n}}\right]$ for the subsimplex of $\Delta^{\left\{m_{0}, \ldots, m_{k}\right\}}$ having vertices $m_{j_{0}}, \ldots, m_{j_{n}}$.

\section{A quick introduction to quasi-categories}

In this section we give an account of the basic properties of quasi-categories, including the Joyal model structure. Almost all of this material is due to Joyal [15], and is also buried deep in [16]. A few simple proofs are included here, but the longer proofs are not. The reader is referred to the appendices for a guide through these longer proofs.

\subsection{Isomorphisms in quasi-categories}

For a quasi-category $X$, we refer to the 0 -simplices $a \in X_{0}$ as objects; to the $1-$ simplices $f \in X_{1}$ as morphisms, which we denote by $f: a \rightarrow b$ if $d_{1}(f)=a$ and $d_{0}(f)=b$; and to the degenerate 1 -simplices $s_{0}(a)$ as identity morphisms, which we denote by $\operatorname{id}_{a}$.

For any set $S$, let $E S$ be the 0 -coskeleton of the set $S$. Write $E^{n}$ for $E(\{0,1, \ldots, n\})$. The $n$-simplices of $E^{1}$ are strings of 0 's and 1 's of length $n+1$, with the face and degeneracy maps coinciding with omission and repetition of entries. Note that $E^{1}$ has exactly two nondegenerate simplices in each dimension, and that its geometric realization is the usual model for $S^{\infty}$. See [8, Section 2.3] for more about the functor $E$.

Let $X$ be a quasi-category. An $\infty$-isomorphism in $X$ is a map $E^{1} \rightarrow X$. We sometimes abuse terminology and say that a 1 -simplex $\Delta^{1} \rightarrow X$ is an $\infty$-isomorphism if it extends to a map $E^{1} \rightarrow X$. 
There is a closely related concept called quasi-isomorphism, which Joyal uses extensively in [15]. This notion at first appears weaker than that of $\infty$-isomorphism, and is typically easier to verify. A quasi-isomorphism is a map $\operatorname{sk}_{2}\left(E^{1}\right) \rightarrow X$. The simplicial set $\operatorname{sk}_{2}\left(E^{1}\right)$ consists of two nondegenerate 1 -simplices and two nondegenerate $2-$ simplices. In other words, a quasi-isomorphism in $X$ consists of two objects $a$ and $b$, maps $f: a \rightarrow b$ and $g: b \rightarrow a$, and two 2-simplices of the form
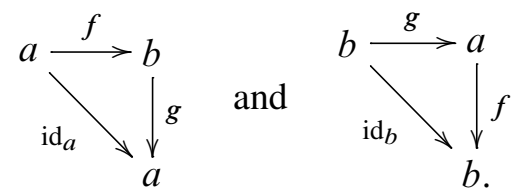

Again, we sometimes abuse terminology and say that a $1-\operatorname{simplex}$ in $X$ is a quasiisomorphism if it extends to a map $\operatorname{sk}_{2}\left(E^{1}\right) \rightarrow X$.

The following results record the key properties we will need about $\infty$-isomorphisms and quasi-isomorphisms:

2.2 Proposition Let $X$ be a quasi-category, and let $f: \Delta^{1} \rightarrow X$. The following conditions on $f$ are equivalent:

(i) $f$ is an $\infty$-isomorphism.

(ii) $f$ is a quasi-isomorphism.

(iii) $f$ has a left quasi-inverse and a (possibly different) right quasi-inverse. That is, there exist 2-simplices in $X$ of the form
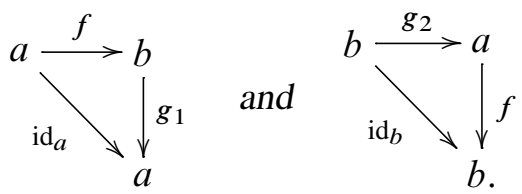

Proof See the proof of Proposition 2.2 in Appendix B.

2.3 Proposition Let $X$ be a quasi-category. Then the map $X \rightarrow *$ has the rightlifting-property with respect to the following:

(a) The maps $\left(A \times E^{1}\right) \amalg_{(A \times\{0\})}(B \times\{0\}) \hookrightarrow B \times E^{1}$, for every monomorphism $A \hookrightarrow B$.

(b) The inclusion $E(\{0,1\}) \cup E(\{1,2\}) \hookrightarrow E(\{0,1,2\})$.

Note that part (b) implies that in a quasi-category one may compose (in a nonunique way) $\infty$-isomorphisms to yield another $\infty$-isomorphism. 
Proof Part (a) is a combinatorial argument which we postpone (see Appendix B). However, (b) is an easy consequence of (a), using the maps $j: E^{2} \rightarrow E^{1} \times E^{1}$ and $p: E^{1} \times E^{1} \rightarrow E^{2}$ defined by

$$
j(0)=(0,0), \quad j(1)=(1,0), \quad j(2)=(1,1)
$$

and

$$
p(0,0)=p(0,1)=0, \quad p(1,0)=1, \quad p(1,1)=2 .
$$

(Both $E^{2}$ and $E^{1} \times E^{1}$ are 0 -coskeleta, so a map into them is completely determined by what it does to 0 -simplices). The maps $j$ and $p$ exhibit the inclusion from (b) as a retract of $\left(\{0,1\} \times E^{1}\right) \amalg_{\{0,1\} \times\{0\}}\left(E^{1} \times\{0\}\right) \hookrightarrow E^{1} \times E^{1}$. Since $X \rightarrow *$ has the right-lifting-property with respect to the latter map, it also has this property with respect to any retract.

The proofs of Propositions 2.2 and 2.3(a) are not at all straightforward. They hinge on a lemma discovered by Joyal which we also record here (although the proof is again deferred to Appendix B).

2.4 Proposition (Joyal, Special outer horn lifting) Let $X$ be a quasi-category. Given either of the following lifting diagrams
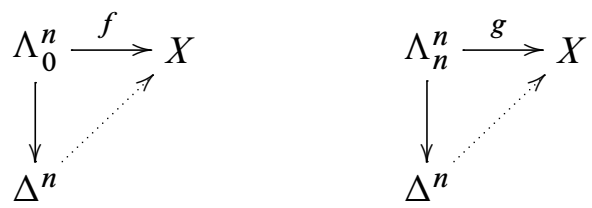

in which, respectively, $f([0,1])$ or $g([n-1, n])$ is a quasi-isomorphism, there is a lifting as shown.

It is easy to see that in a Kan complex every morphism is a quasi-isomorphism (use Proposition 2.2). Conversely, if in a certain quasi-category $X$ every morphism is a quasi-isomorphism then Proposition 2.4 implies that $X$ is a Kan complex. Thus one has the slogan "Kan complexes are $\infty$-groupoids."

\subsection{Box product lemmas}

A map of simplicial sets will be called inner anodyne if it can be constructed out of the maps $\Lambda_{k}^{n} \hookrightarrow \Delta^{n}, 0<k<n$, by cobase changes and compositions. Clearly any quasi-category has the right-lifting-property with respect to all inner anodyne maps.

Given morphisms $f: A \rightarrow B$ and $g: C \rightarrow D$, let $f \square g$ denote the induced map

$$
(A \times D) \amalg_{(A \times C)}(B \times C) \longrightarrow B \times D .
$$


2.6 Proposition (Joyal) If $i: \Lambda_{k}^{n} \hookrightarrow \Delta^{n}$ is an inner horn inclusion and the map $j: A \rightarrow B$ is any monomorphism, then $i \square j$ is inner anodyne.

Proof See Appendix A.

A map $X \rightarrow Y$ is an inner fibration if it has the right-lifting-property with respect to the inner horn inclusions. The following result is an immediate consequence of Proposition 2.6, using adjointness:

2.7 Proposition If $A \succ B$ is a monomorphism and $X \rightarrow Y$ is an inner fibration, then $X^{B} \rightarrow X^{A} \times_{Y^{A}} Y^{B}$ is also an inner fibration. In particular, if $X$ is a quasi-category then so is $X^{A}$ for any simplicial set $A$.

Proof Follows immediately from Proposition 2.6.

\subsection{Joyal equivalences}

Let $A$ and $Z$ be simplicial sets, and assume $Z$ is a quasi-category. Define a relation on ${ }_{s} \operatorname{Set}(A, Z)$ by saying that $f$ and $g$ are $E^{1}$-homotopic, denoted $f \sim g$, if there exists a map $H: A \times E^{1} \rightarrow Z$ such that $H i_{0}=f$ and $H i_{1}=g$, where $i_{0}, i_{1}: A \hookrightarrow A \times E^{1}$ are the evident inclusions. It is easy to see that this relation is both reflexive and symmetric, and Proposition 2.3(b) implies that it is also transitive (using that $Z^{A}$ is a quasi-category). Thus $\sim$ is an equivalence relation and we write $[A, Z]_{E^{1}}$ to denote the set $s \mathscr{S e t}(A, Z) / \sim$.

2.9 Definition A map of simplicial sets $A \rightarrow B$ is a Joyal equivalence if $[B, Z]_{E^{1}} \rightarrow$ $[A, Z]_{E^{1}}$ is a bijection for every quasi-category $Z$. A Joyal acyclic cofibration is a map which is both a monomorphism and a Joyal equivalence.

2.10 Remark We define a map of simplicial sets $f: X \rightarrow Y$ to be an $E^{1}$-homotopy equivalence if there is a map $g: Y \rightarrow X$ such that $f g$ and $g f$ are $E^{1}$-homotopic to their respective identities. It is trivial to check that every $E^{1}$-homotopy equivalence is a Joyal equivalence, and that if $X$ and $Y$ are quasi-categories then a map $X \rightarrow Y$ is a Joyal equivalence if and only if it is an $E^{1}$-homotopy equivalence. Note that $\{0\} \hookrightarrow E^{1}$ is readily seen to be an $E^{1}$-homotopy equivalence.

2.11 Proposition The following statements are true:

(a) If $C \rightarrow D$ is a Joyal acyclic cofibration then so is $A \times C \rightarrow A \times D$, for any simplicial set $A$.

(b) If $X$ is a quasi-category, then $X \rightarrow \Delta^{0}$ has the right-lifting-property with respect to every Joyal acyclic cofibration.

(c) Every inner horn inclusion $\Lambda_{k}^{n} \hookrightarrow \Delta^{n}, 0<k<n$, is a Joyal equivalence. 
Proof For (a) we must show that $[A \times D, X]_{E^{1}} \rightarrow[A \times C, X]_{E^{1}}$ is a bijection for every quasi-category $X$. But this map is readily identified with $\left[D, X^{A}\right]_{E^{1}} \rightarrow\left[C, X^{A}\right]_{E^{1}}$, which is a bijection because $X^{A}$ is also a quasi-category (Proposition 2.7).

For part (b) we begin with a commutative square

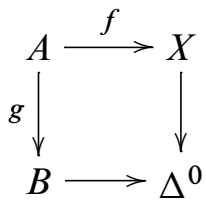

in which $g$ is a Joyal acyclic cofibration. Since $[B, X]_{E^{1}} \rightarrow[A, X]_{E^{1}}$ is bijective, there is a map $h: B \rightarrow X$ and an $E^{1}$-homotopy between $h g$ and $f$. But this then yields a diagram

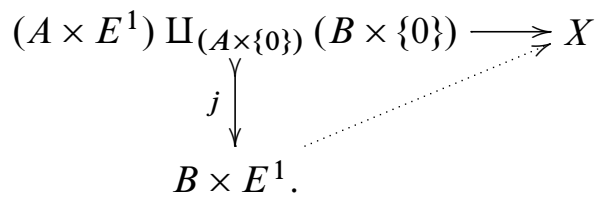

By Proposition 2.3(a) an extension exists as shown. Restricting to $B \times\{1\}$ gives the desired lift for our original square.

For part (c), if $X$ is a quasi-category then $\left[\Delta^{n}, X\right]_{E^{1}} \rightarrow\left[\Lambda_{k}^{n}, X\right]_{E^{1}}$ is surjective by the definition of quasi-category. Injectivity follows from Proposition 2.6, which says that $X \rightarrow *$ has the RLP with respect to $\left(\Lambda_{k}^{n} \hookrightarrow \Delta^{n}\right) \square\left(\{0,1\} \hookrightarrow E^{1}\right)$.

\subsection{The model category structure}

Like all the results in this section, the following is due to Joyal. It is proven in Appendix C.

2.13 Theorem There exists a unique model structure on $s$ Set in which the cofibrations are the monomorphisms and the fibrant objects are the quasi-categories. The weak equivalences in this structure are the Joyal equivalences, and the model structure is cofibrantly-generated.

The model category structure provided by Theorem 2.13 will be denoted $s \operatorname{Set}_{J}$. Note that it is left proper because every object is cofibrant. We will use the term Joyal fibration for the fibrations in $s \mathscr{S e t}_{J}$.

2.14 Remark It should be noted that while $s \operatorname{Set}_{J}$ is cofibrantly-generated, no one has so far managed to write down a manageable set of generating acyclic cofibrations. 
The following result will be used frequently:

2.15 Proposition Let $f: A \succ B$ and $g: C \succ D$ be cofibrations in $s$ Set $_{J}$, and let $h: X \rightarrow Y$ be a Joyal fibration. Then the following statements are true:

(a) $f \square g$ is a cofibration, which is Joyal acyclic if $f$ or $g$ is so.

(b) $X^{B} \rightarrow\left(Y^{B} \times_{Y_{A}} X^{A}\right)$ is a Joyal fibration, which is acyclic if $f$ or $h$ is so.

Proof Part (b) follows immediately from (a) by adjointness. The statement in (a) that $f \square g$ is a cofibration is evident, so we must only prove that it is a Joyal equivalence if $f$ is (the case when $g$ is a Joyal equivalence following by symmetry). Consider the diagram

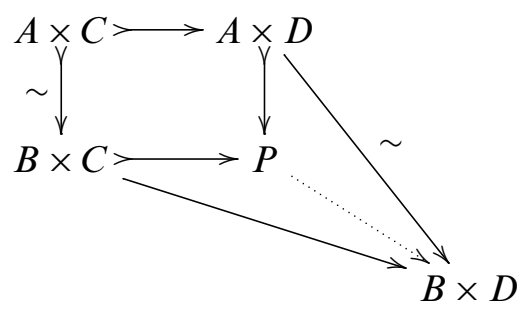

where $P$ is the pushout. The labelled maps are Joyal equivalences by Proposition 2.11(a). It follows that the pushout map $A \times D \rightarrow P$ is a Joyal acyclic cofibration, and hence $P \rightarrow B \times D$ is a Joyal equivalence by two-out-of-three.

\subsection{Rigidification and the coherent nerve}

We recall from [16, Section 1.1.5] that there are adjoint functors

$$
\mathfrak{C}: s \mathscr{S e t} \rightleftarrows s \text { bat }: N \text {. }
$$

Here $\mathfrak{C}\left(\Delta^{n}\right)$ is a simplicial category which one explicitly constructs, and $N(\mathscr{D})$ is the simplicial set $[n] \mapsto s \mathscr{C a t}\left(\mathfrak{C}\left(\Delta^{n}\right)\right.$, D). See [8] for more information, as well for a very detailed description of the functor $\mathfrak{C}$. We call $\mathfrak{C}$ the rigidification functor, and $N$ the coherent nerve.

It is useful to also consider the composite of adjoint pairs

$$
\text { s Set } \stackrel{\mathfrak{C}}{\underset{N}{\gtrless}} s^{C} \text { at } \stackrel{\pi_{0}}{\underset{c}{\gtrless}} \mathscr{C} \text { at. }
$$

where the left adjoints all point left to right. Here $c$ is the functor which regards a category as a discrete simplicial category, and $\pi_{0}$ is the functor which applies the usual $\pi_{0}$ to all the mapping spaces. Note that the composite $N c$ is the classical 
nerve functor; as $\pi_{0} \mathfrak{C}$ is its left adjoint, this identifies $\pi_{0} \mathfrak{C}$ as the functor which takes a simplicial set and forms the free category from the 0 -simplices and 1 -simplices, imposing composition relations coming from the 2 -simplices. This functor is denoted $\tau_{1}$ in [15], but we will always call it $\pi_{0} \mathfrak{C}$ in the present paper.

2.17 Remark For a general simplicial set $X$, a map in $\pi_{0} \mathfrak{C}(X)$ from $a$ to $b$ is a formal composite of maps represented by 1 -simplices in $X$. But in a quasi-category we may "compose" adjacent 1-simplices by filling an inner horn of dimension 2 , and this shows that every map in $\pi_{0} \mathfrak{C}(X)$ is represented by a single 1 -simplex of $X$.

The following result is due to Joyal:

2.18 Proposition Let $X$ be a quasi-category, and let $f, g$, and $h$ be 1-simplices in $X$. Then one has $h=g \circ f$ in $\pi_{0} \mathfrak{C}(X)$ if and only if there exists a map $\Delta^{2} \rightarrow X$ whose $0-, 1-$ and 2 -faces are $g, h$ and $f$, respectively.

The proof will be given in Appendix B. The following is an immediate corollary:

2.19 Corollary Let $f: \Delta^{1} \rightarrow X$ be a 1 -simplex in a quasi-category $X$. Then $f$ is a quasi-isomorphism if and only if the image of $f$ in the category $\pi_{0} \mathfrak{C}(X)$ is an isomorphism.

Proof Immediate from Proposition 2.18.

Note that Corollary 2.19 adds another equivalent condition to the list given in Proposition 2.2. Also observe that this corollary implies a two-out-of-three property for quasiisomorphisms: for a 2-simplex in $X$, if two of its three faces are quasi-isomorphisms then so is the third.

We now also have the following:

2.20 Proposition For $X$ a quasi-category, there is a natural bijection between $[*, X]_{E^{1}}$ and the set of isomorphism classes of objects in $\pi_{0} \mathfrak{C}(X)$, denoted iso $\left(\pi_{0} \mathfrak{C}(X)\right)$.

Proof First note that $\pi_{0} \mathfrak{C}\left(E^{1}\right)$ is the category consisting of two objects and a unique isomorphism between them. We have the following map of coequalizer diagrams:

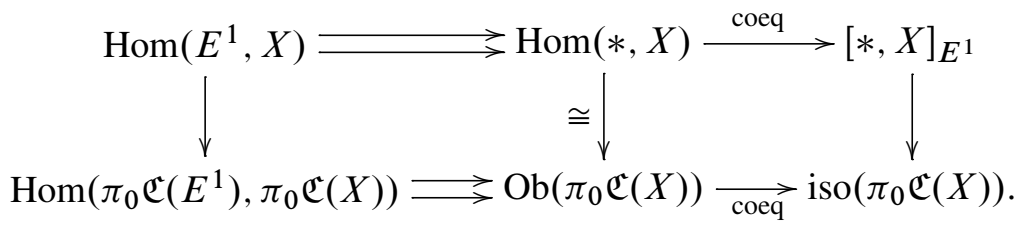


The right-hand vertical map is surjective because the middle map is a bijection; we need to show that it is injective.

Suppose two 0 -simplices $a, b \in X_{0}$ are isomorphic in $\pi_{0} \mathfrak{C}(X)$. There is a 1 -simplex $e$ in $X$ connecting $a$ and $b$ that represents this isomorphism, and by Corollary 2.19, $e$ is also a quasi-isomorphism. By Proposition 2.2, $e$ extends to a map $E^{1} \rightarrow X$. Thus $a$ and $b$ are equal in $[*, X]_{E^{1}}$, as desired.

\section{Background on mapping spaces in model categories}

Given two objects $X$ and $Y$ in a model category $\mathcal{M}$, there is an associated simplicial set $\operatorname{hMap}_{\mathcal{M}}(X, Y)$ called a "homotopy function complex" from $X$ to $Y$. The basic theory of these function complexes is due to Dwyer and Kan $[11 ; 9 ; 10]$. As recounted in the introduction, there are several different ways to write down models for these function complexes, all of which turn out to be Kan equivalent. In this section we give a brief review of some of this machinery.

\subsection{Mapping spaces via cosimplicial resolutions}

Let $M$ be a model category, and let $c . M$ be the Reedy model category of cosimplicial objects in $\mathcal{M}$ [12, Chapter 15]. For any $X \in \mathcal{M}$ we will write $c X$ for the constant cosimplicial object consisting of $X$ in every dimension, where every coface and codegeneracy is the identity.

If $X \in \mathcal{M}$, a cosimplicial resolution of $X$ is a Reedy cofibrant replacement $Q^{\bullet} \stackrel{\sim}{\longrightarrow} c X$. Given such a cosimplicial resolution and an object $Z \in \mathcal{M}$, we may form the simplicial set $M\left(Q^{\bullet}, Z\right)$ given by

$$
[n] \mapsto M\left(Q^{n}, Z\right) .
$$

It is known [12, 16.5.5] that if $Z \rightarrow Z^{\prime}$ is a weak equivalence between fibrant objects then the induced map $\mathcal{M}\left(Q^{\bullet}, Z\right) \rightarrow \mathcal{M}\left(Q^{\bullet}, Z^{\prime}\right)$ is a Kan equivalence of simplicial sets.

\subsection{Mapping spaces via nerves of categories}

For any object $X \in M$, let $2(X)$ be the category whose objects are pairs $[Q, Q \rightarrow X]$ where $Q$ is cofibrant and $Q \rightarrow X$ is a weak equivalence. For any object $Y \in \mathcal{M}$, there is a functor

$$
M(-, Y): 2(X)^{\mathrm{op}} \longrightarrow \mathscr{S e t}
$$

sending $[Q, Q \rightarrow X]$ to $\mu(Q, Y)$. We can regard this functor as taking values in $s \mathscr{S}$ et by composing with the embedding $\mathscr{S e t} \hookrightarrow s$ Set. 
Consider the simplicial set hocolim $2(X)$ op $M(-, Y)$. We fix our model for the hocolim functor to be the result of first taking the simplicial replacement of a diagram (cf Bousfield and Kan [4, XII.5]) and then applying geometric realization. Notice in our case that in dimension $n$ the simplicial replacement consists of diagrams of weak equivalences $Q_{0} \leftarrow Q_{1} \leftarrow \ldots \sim Q_{n}$ over $X$ (with each $Q_{i} \rightarrow X$ in $2(X)$ ), together with a map $Q_{0} \rightarrow Y$. This shows that the simplicial replacement is nothing but the nerve of the category for which an object is a zigzag $[X \leftarrow Q \rightarrow Y]$, where $Q$ is cofibrant and $Q \rightarrow X$ is a weak equivalence; and a map from $[X \leftarrow Q \rightarrow Y]$ to $\left[X \leftarrow Q^{\prime} \rightarrow Y\right]$ is a map $Q^{\prime} \rightarrow Q$ making the evident diagram commute.

Categories of zigzags like the one considered above were first studied in [10]. There are many variations, and it is basically the case that all sensible variations have Kan equivalent nerves; moreover, these nerves are Kan equivalent to the homotopy function complex $\operatorname{hMap}_{\mathcal{M}}(X, Y)$ (defined to be the space of maps in the simplicial localization of $\mathcal{M}$ with respect to the weak equivalences). We will next recall some of this machinery. In addition to [10], see [6].

Following [10], write (Wcofib) $)^{-1} M(\mathrm{Wfib})^{-1}(X, Y)$ to denote the category whose objects are zigzags

$$
X \stackrel{\sim}{\longleftarrow} U \longrightarrow V \stackrel{\sim}{\longleftarrow} Y,
$$

and where the maps are natural transformations of diagrams which are the identity on $X$ and on $Y$. Similarly, let $W^{-1} \mathcal{M} W^{-1}(X, Y)$ be the category whose objects are zigzags

$$
X \stackrel{\sim}{\longleftarrow} U \longrightarrow V \stackrel{\sim}{\longleftarrow} Y,
$$

let $M(\text { Wfib })^{-1} \mathcal{M}(X, Y)$ be the category whose objects are zigzags

$$
X \longrightarrow U \stackrel{\sim}{\longleftarrow} V \longrightarrow Y,
$$

and so on.

Observe that there are natural inclusions of two types: an example of the first is $\mathcal{M}(\mathrm{Wfib})^{-1}(X, Y) \hookrightarrow \mathcal{M \mathrm { W } ^ { - 1 }}(X, Y)$ (induced by Wfib $\hookrightarrow \mathrm{W}$ ), and an example of the second is

$$
\mu \mathrm{W}^{-1}(X, Y) \hookrightarrow \mu \mathrm{W}^{-1} \mathcal{M}(X, Y)
$$

which sends

$$
[X \stackrel{\sim}{\longleftarrow} A \longrightarrow Y] \mapsto[X \stackrel{\text { id }}{\longrightarrow} X \stackrel{\sim}{\longleftarrow} A \longrightarrow Y]
$$

The following proposition is a very basic one in this theory, and will be used often in the remainder of the paper; we have included the proof for completeness, and because it is simple. 
3.3 Proposition When $Y$ is fibrant, the maps in the following commutative square all induce Kan equivalences on nerves:

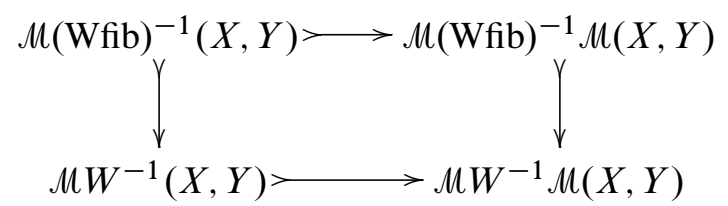

Proof We start with the left vertical map, which we denote $j_{1}$. Given a zigzag $[X \leftarrow A \rightarrow Y$ ], functorially factor the map $A \rightarrow X \times Y$ as $A \stackrel{\sim}{\longrightarrow} P \rightarrow X \times Y$. Since $Y$ is fibrant the projection $X \times Y \rightarrow X$ is a fibration, and so the composite $P \rightarrow X$ is a fibration as well. Define a functor $F: \mu \mathrm{W}^{-1}(X, Y) \rightarrow \mathcal{M}(\mathrm{Wfib})^{-1}(X, Y)$ by sending

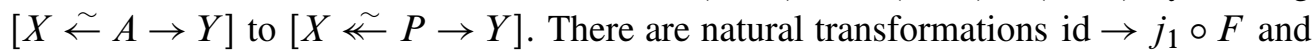
id $\rightarrow F \circ j_{1}$, which shows that on nerves $F$ and $j_{1}$ are homotopy inverses.

A very similar proof works for the right vertical map in the diagram, which we'll denote $j_{2}$. Given a zigzag $[X \rightarrow U \stackrel{\leftarrow}{\leftarrow} \rightarrow Y$ ], functorially factor $V \rightarrow U \times Y$ as $V \stackrel{\sim}{\rightarrow} P \rightarrow U \times Y$. Define $F: M W^{-1} \mathcal{M}(X, Y) \rightarrow \mathcal{M}(\mathrm{Wfib})^{-1} \mathcal{M}(X, Y)$ by

$$
[X \longrightarrow U \stackrel{\sim}{\longleftarrow} V \longrightarrow Y] \mapsto[X \longrightarrow U \stackrel{\sim}{\longleftarrow} P \longrightarrow Y] .
$$

This gives a homotopy inverse for $j_{2}$.

For the top horizontal map we do not even need to use that $Y$ is fibrant. Define a homotopy inverse by sending $[X \rightarrow U \leftarrow V \rightarrow Y]$ to the associated zigzag $[X \stackrel{\leftarrow}{\leftarrow} \rightarrow \rightarrow$ ] where $P$ is the pullback of $X \rightarrow U \leftarrow V$.

Finally, the bottom horizontal map induces a Kan equivalence on nerves because the other three maps do.

Let $Q X^{\bullet} \rightarrow X$ be a cosimplicial resolution of $X$ in $c \mathcal{M}$. Following [10], we now relate the simplicial set $M\left(Q X^{\bullet}, Y\right)$ to the nerves of the categories of zigzags considered above.

For any simplicial set $K$, let $\Delta K$ be the category of simplices of $K$. This is none other than the overcategory $(S \downarrow K)$, where $S: \Delta \rightarrow s \mathscr{S}$ et is the functor $[n] \mapsto \Delta^{n}$. It is known that the nerve of $\Delta K$ is naturally Kan equivalent to $K$ (see [6, text prior to Theorem 2.4] for an explanation).

There is a functor $\Delta M\left(Q X^{\bullet}, Y\right) \rightarrow \mathcal{M}(\mathrm{Wfib})^{-1}(X, Y)$ sending $\left([n], Q X^{n} \rightarrow Y\right)$ to $\left[X \stackrel{\sim}{\leftarrow} Q X^{n} \rightarrow Y\right]$.

3.4 Proposition Let $Q X^{\bullet} \rightarrow X$ be a Reedy cofibrant resolution of $X$. Then $\Delta \mu\left(Q X^{\bullet}, Y\right) \rightarrow M(\text { Wfib })^{-1}(X, Y)$ induces a Kan equivalence on nerves.

Proof The result is proven in [10], but see also [6, Theorem 2.4]. 
3.5 Remark To briefly summarize the main points of this section, Proposition 3.3 and Proposition 3.4 show that when $X$ is any object and $Y$ is a fibrant object in a model category $\mathcal{M}$, the following maps of categories all induce Kan equivalences on the nerves:

$$
\Delta M\left(Q X^{\bullet}, Y\right) \longrightarrow \mu(\mathrm{Wfib})^{-1}(X, Y)>\mathrm{MW}^{-1}(X, Y)>\mathrm{NW}^{-1} \mathcal{M}(X, Y) .
$$

In particular, the nerves all have the homotopy type of $\mathcal{M}\left(Q X^{\bullet}, Y\right)$, which is a model for the homotopy function complex $\operatorname{hMap}(X, Y)$.

\section{Dwyer-Kan models for quasi-category mapping spaces}

In this section we use the Dwyer-Kan machinery of the previous section to give models for the mapping spaces in a quasi-category. These models have the advantage of being relatively easy to work with and compute. However, they have the disadvantage that they do not admit a composition law.

\subsection{The canonical cosimplicial framing on $s \mathscr{S e t}_{J}$}

Recall from Section 2 that $E:$ Yet $\rightarrow s$ Set denotes the 0 -coskeleton functor. For a set $S$, we may also describe $E S$ as the nerve of the groupoid $E_{G} S$ with object set $S$ and a single morphism $a \rightarrow b$ for each $a, b \in S$.

Recall the Reedy model structure [12, Chapter 15.3] on the category of cosimplicial objects in a model category. In the present section, the Reedy structure we will use will always be on $c\left(s \operatorname{Set}_{J}\right)$, not $c\left(s \operatorname{Set}_{K}\right)$. However, note that any Reedy-Joyal equivalence is also a Reedy-Kan equivalence.

4.2 Lemma For any nonempty set $S$, the map $E S \rightarrow \Delta^{0}$ is an acyclic fibration in $s \operatorname{Set}_{J}$.

Proof The acyclic fibrations in the Joyal model category $s \mathscr{S}_{e} t_{J}$ are the same as those in the Kan model category $s \mathscr{S e t}_{K}$, as both model categories have the same cofibrations. It is easy to check that $E S \rightarrow \Delta^{0}$ has the right lifting property with respect to the maps $\partial \Delta^{n} \rightarrow \Delta^{n}$.

The forgetful functor $\Delta \hookrightarrow$ Yet describes a cosimplicial set whose $n$-th object is $[n]=\{0,1, \ldots, n\}$. Applying the functor $E$ gives a cosimplicial object $[n] \mapsto E^{n}=$ $N\left(E_{G}([n])\right)$ in $s \mathscr{S e t}_{J}$. It is easy to check that $E^{\bullet}$ is Reedy cofibrant in $c\left(s \operatorname{Set}_{J}\right)$ (use [12, Corollary 15.9.10], for example). Also, the above lemma shows that each $E^{n}$ is 
contractible in $s \mathscr{S e t}_{J}$. Note that the evident inclusion of categories $[n] \hookrightarrow E_{G}([n])$ induces a levelwise cofibration $\Delta^{\bullet} \hookrightarrow E^{\bullet}$, which in each level is a Kan equivalence but not a Joyal equivalence.

For any simplicial set $X$, the cosimplicial object $[n] \mapsto X \times E^{n}$ is a cosimplicial resolution of $X$ with respect to the model structure $s \mathscr{S e t}_{J}$.

\subsection{Three cosimplicial versions of $\Delta^{1}$}

In his book, Lurie at various times uses three internal models for the mapping space between vertices in a simplicial set $S$. They are called $\operatorname{Hom}_{S}^{R}, \operatorname{Hom}_{S}^{L}$, and $\operatorname{Hom}_{S}$; the descriptions of all of these can be found in [16, Section 1.2.2]. In this subsection we show that each of these can be understood as the mapping space coming from a cosimplicial resolution of $\Delta^{1}$. We also give one new model, $\operatorname{Hom}_{S}^{E}$, which will be very useful later. See Remark 4.9 for more about the Lurie models.

Recall that for any simplicial sets $M$ and $N$, the join $M \star N$ is a simplicial set with

$$
(M \star N)_{n}=\coprod_{-1 \leq i \leq n} M_{i} \times N_{n-i-1},
$$

where we put $M_{-1}=N_{-1}=\Delta^{0}$ (see $[16,1.2 .8 .1]$ ). Note that $\star$ is a bifunctor and that $M \star \varnothing=\varnothing \star M=M$, so in particular there are natural inclusions $M \hookrightarrow M \star N$ and $N \hookrightarrow M \star N$. Note as well that $M \star \Delta^{0}$ and $\Delta^{0} \star M$ are cones on $M$, and that $\Delta^{n} \star \Delta^{r} \cong \Delta^{n+r+1}$.

We let $C_{R}(M)$ and $C_{L}(M)$ denote the quotient $\left(M \star \Delta^{0}\right) / M$ and $\left(\Delta^{0} \star M\right) / M$, respectively. For any simplicial set $M$, let $C_{\text {cyl }}(M)$ be the pushout

$$
\partial \Delta^{1} \leftarrow M \times \partial \Delta^{1} \hookrightarrow M \times \Delta^{1} .
$$

Note that each of $C_{R}(M), C_{L}(M)$, and $C_{\text {cyl }}(M)$ has exactly two 0 -simplices and comes with a natural map to $\Delta^{1}$ (which is unique over $\partial \Delta^{1}$ ).

Let $C_{R}^{\bullet}$ (respectively $C_{L}^{\bullet}$ ) denote the cosimplicial space $[n] \mapsto C_{R}\left(\Delta^{n}\right)$ (resp. $[n] \mapsto$ $C_{L}\left(\Delta^{n}\right)$ ). Write $C_{\text {cyl }}^{\bullet}$ for the cosimplicial space $[n] \mapsto C_{\text {cyl }}\left(\Delta^{n}\right)$. Finally, write $C_{E}^{\bullet}$ for the cosimplicial space $[n] \mapsto C_{\text {cyl }}\left(E^{n}\right)$.

Note that there are inclusions $C_{R}\left(\Delta^{n}\right) \hookrightarrow C_{\text {cyl }}\left(\Delta^{n}\right)$, and surjection maps $C_{\text {cyl }}\left(\Delta^{n}\right) \rightarrow$ $C_{R}\left(\Delta^{n}\right)$ exhibiting $C_{R}\left(\Delta^{n}\right)$ as a retract of $C_{\text {cyl }}\left(\Delta^{n}\right)$. These assemble to give maps of cosimplicial spaces (in both directions) between $C_{R}^{\bullet}$ and $C_{\text {cyl }}^{\bullet}$. The same can be said if we replace $C_{R}$ with $C_{L}$. In addition, the map of cosimplicial spaces $\Delta^{\bullet} \rightarrow E^{\bullet}$ gives 
us a map $C_{\mathrm{cyl}}^{\bullet} \rightarrow C_{E}^{\bullet}$. In other words, we have maps

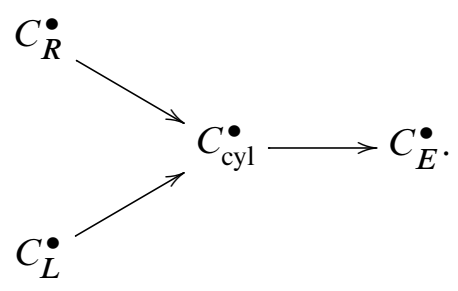

Note that in each of these cosimplicial spaces the 0 -th space is $\Delta^{1}$. Hence, each of the above four cosimplicial spaces comes equipped with a canonical map to $c \Delta^{1}$. Each also comes equipped with a canonical map from $c\left(\partial \Delta^{1}\right)$.

Recall the notation $s \mathscr{S e t}_{*, *}=\left(\partial \Delta^{1} \downarrow s \mathscr{S e t}_{J}\right)$, and let $c\left(s \mathscr{S e t}_{*, *}\right)$ denote the Reedy model structure on the cosimplicial objects in $s \mathscr{S e t}_{*, *}$, as in Section 3.

\subsection{Proposition}

(a) Each of the maps $c\left(\partial \Delta^{1}\right) \rightarrow C_{R}^{\bullet}, c\left(\partial \Delta^{1}\right) \rightarrow C_{L}^{\bullet}, c\left(\partial \Delta^{1}\right) \rightarrow C_{\text {cyl }}^{\bullet}$ and $c\left(\partial \Delta^{1}\right) \rightarrow$ $C_{E}^{\bullet}$ is a Reedy cofibration.

(b) Each of $C_{R}^{\bullet}, C_{L}^{\bullet}, C_{\text {cyl }}^{\bullet}$ and $C_{E}^{\bullet}$ is Reedy cofibrant as an object of $c\left(s \operatorname{Set}_{*, *}\right)$.

(c) Each of the maps of simplicial sets $C_{R}^{n} \rightarrow \Delta^{1}, C_{L}^{n} \rightarrow \Delta^{1}, C_{\mathrm{cyl}}^{n} \rightarrow \Delta^{1}$ and $C_{E}^{n} \rightarrow \Delta^{1}$ is a Joyal equivalence.

(d) Consequently, each of $C_{R}^{\bullet}, C_{L}^{\bullet}, C_{\text {cyl }}^{\bullet}$ and $C_{E}^{\bullet}$ is a cosimplicial resolution of $\Delta^{1}$ in $c\left(s \mathscr{S e t}_{*, *}\right)$.

Proof Parts (a) and (b) are obvious, and (d) follows immediately from (b) and (c). For (c), note first that $C_{E}^{n} \rightarrow \Delta^{1}$ is easily seen to be a Joyal equivalence. Indeed, $C_{E}^{n}$ is the pushout of

$$
\partial \Delta^{1} \stackrel{\sim}{\leftarrow} \Delta^{1} \times E^{n} \hookrightarrow \Delta^{1} \times E^{n},
$$

where the indicated map is a Joyal equivalence by Lemma 4.2. It follows from left properness of $s \mathscr{S e t}_{J}$ that $\Delta^{1} \times E^{n} \rightarrow C_{E}^{n}$ is a Joyal equivalence. Using that $\Delta^{1} \times E^{n} \rightarrow \Delta^{1}$ is a Joyal equivalence (Lemma 4.2 again), it follows immediately that $C_{E}^{n} \rightarrow \Delta^{1}$ is also one.

The arguments for $C_{R}^{n}, C_{L}^{n}$ and $C_{\mathrm{cyl}}^{n}$ are more complicated. Picking the former for concreteness, there are various sections of the map $C_{R}^{n} \rightarrow \Delta^{1}$. It will be sufficient to show that any one of these is a Joyal acyclic cofibration, which we do by exhibiting it as a composition of cobase changes of inner horn inclusions. This is not difficult, but it is a little cumbersome; we postpone the proof until Section 9 (see Proposition 9.4). 


\subsection{Application to mapping spaces}

For every $S \in s_{S e t_{J}}$ and every $a, b \in S$, define $\operatorname{Hom}_{S}^{R}(a, b)$ to be the simplicial set ${ }_{s} \operatorname{Set}_{*, *}\left(C_{R}^{\bullet}, S_{a, b}\right)$. Note that this is also the pullback of

$$
* \stackrel{(a, b)}{\longrightarrow} S \times S \ll s \mathscr{e} \operatorname{et}\left(C_{R}^{\bullet}, S\right) .
$$

Define $\operatorname{Hom}_{S}^{L}(a, b), \operatorname{Hom}_{S}^{\mathrm{cyl}}(a, b)$, and $\operatorname{Hom}_{S}^{E}(a, b)$ analogously, and note that diagram (4.4) induces natural maps

$$
\operatorname{Hom}_{S}^{E}(a, b) \longrightarrow \operatorname{Hom}_{S}^{\mathrm{cyl}}(a, b) \longrightarrow \operatorname{Hom}_{S}^{R}(a, b)
$$

4.8 Corollary When $S \in s_{\text {Set }}$ is fibrant and $a, b \in S$, the four natural maps in (4.7) are Kan equivalences of simplicial sets. These simplicial sets are models for the homotopy function complex hMap $\operatorname{het}_{*, *}\left(\Delta^{1}, S_{a, b}\right)$.

Proof This is immediate from Proposition 4.5 and $[12,16.5 .5]$.

4.9 Remark For a simplicial set $S$ and vertices $a, b \in S_{0}$, our notation $\operatorname{Hom}_{S}^{R}(a, b)$, $\operatorname{Hom}_{S}^{L}(a, b)$ agrees with that of [16, Section 1.2.2]. Our notation $\operatorname{Hom}_{S}^{\text {cyl }}(a, b)$ is denoted $\operatorname{Hom}_{S}(a, b)$ in [16], and we used the Lurie notation earlier in Proposition 1.2. Note that $\operatorname{Hom}_{S}^{\text {cyl }}(a, b)$ can also be described as the fiber of the morphism of simplicial mapping spaces $\operatorname{Map}_{s \varphi_{e t}}\left(\Delta^{1}, S\right) \rightarrow \operatorname{Map}_{s \varphi_{e t}}\left(\partial \Delta^{1}, S\right)$ over the point $(a, b)$. The model $\operatorname{Hom}_{S}^{E}(a, b)$ does not seem to appear in [16], but will be very useful in Section 6.

The following calculation will be needed in the next section. Recall the notion of necklace, from [8, Section 3], and that if $T=\Delta^{n_{1}} \vee \cdots \vee \Delta^{n_{k}}$ is a necklace then $\Delta[T]$ denotes the simplex spanned by the ordered set of vertices of $T$.

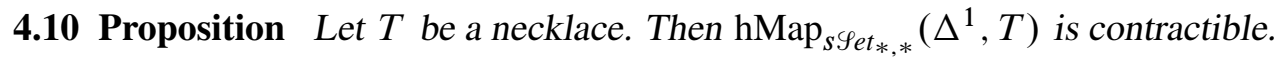

Proof It is a fact that $T \rightarrow \Delta[T]$ is a Joyal equivalence; see Lemma 9.1 for a proof. Also, $\Delta[T]$ is fibrant in $s \mathscr{S e t}_{J}$ because it is the nerve of a category (as is any $\Delta^{k}$ ). We may therefore model our homotopy function complex by

$$
{ }_{s} \mathscr{S e t}_{*, *}\left(C_{R}^{\bullet}, \Delta[T]\right)
$$

where $C_{R}^{\bullet}$ is the cosimplicial resolution of $\Delta^{1}$ considered in this section.

It is easy to check that in $s \mathscr{S e t}_{*, *}$ there is a unique map from $C_{R}^{n}$ to $\Delta[T]$, for each $n$ (it factors through $\left.\Delta^{1}\right)$. Therefore we have $s \operatorname{Set}_{*, *}\left(C_{R}^{\bullet}, \Delta[T]\right)=*$, and this completes the proof. 


\section{Connections with the rigidification mapping spaces}

In this section we prove that for any simplicial set $S$ and any $a, b \in S_{0}$, the rigidification mapping space $\mathfrak{C}(S)(a, b)$ is naturally Kan equivalent to the Dwyer-Kan mapping space $\operatorname{hMap}_{\left(s \varphi_{e t, *}\right)_{J}}\left(\Delta^{1}, S_{a, b}\right)$. As a corollary, we prove that for any simplicial category $\mathscr{D}$ the counit map $\mathfrak{C}(N \mathscr{D}) \rightarrow \mathscr{D}$ is a weak equivalence in $s \mathscr{b}$ at.

If $Y$ is an object in $s \mathscr{S} t_{*, *}$ we will write $\alpha$ and $\omega$ for the images of 0 and 1 under the map $\partial \Delta^{1} \rightarrow Y$. We always use the Joyal model structure on the category $s \mathscr{S e t}_{*, *}=\left(\partial \Delta^{1} \downarrow s \mathscr{S e t}\right)$.

If $S$ is a simplicial set and $a, b \in S$, let us use the notation $\operatorname{hMap}(S)(a, b)$ as shorthand for a homotopy function complex $\operatorname{hMap}_{s \varphi_{e t * *}}\left(\Delta^{1}, S_{a, b}\right)$.

Let $\mathscr{Y}$ denote the full subcategory of $s \mathscr{S e t}_{*, *}$ whose objects are spaces $Y$ such that $\operatorname{hMap}(Y)(\alpha, \omega) \simeq *$ and $\mathfrak{C}(Y)(\alpha, \omega) \simeq *$. Note that 9 contains the category $\mathcal{N e c}$ (the category of necklaces) by Proposition 4.10 and [8, Corollary 3.10]. So clearly $y$ is a category of gadgets in the sense of [8, Definition 5.5]. Let $y_{f}$ denote the full subcategory of $\mathscr{y}$ consisting of those objects which are fibrant in $s \mathscr{S e t}_{J}$. Let $\mathfrak{C}^{\mathfrak{y}}$ and $\mathfrak{C}^{\mathfrak{g}_{f}}$ be the corresponding functors $s \mathscr{S e t} \rightarrow s^{C}$ at, as defined in [8, Section 5.4]. So if $S \in s \mathscr{S}_{e t}$ and $a, b \in S_{0}, \mathfrak{C}^{\mathscr{y}}(S)(a, b)$ is the nerve of the category ( $\mathscr{} \downarrow \downarrow S_{a, b}$ ), and similarly for $\mathfrak{C}^{\mathrm{y}_{f}}(S)(a, b)$.

5.1 Remark The two conditions that define $y$ seem like they should be equivalent, and they are. That is, we will show in Corollary 5.3 that the conditions $\mathfrak{C}(Y)(\alpha, \omega) \simeq *$ and $\operatorname{hMap}(Y)(\alpha, \omega) \simeq *$ are equivalent. However, at the moment we do not know this, so including both conditions in the definition of $y$ is not redundant.

Let $C^{\bullet}$ be a cosimplicial resolution of $\Delta^{1}$ in $s \mathscr{S e t}_{J}$. Let $C^{\bullet} \stackrel{\sim}{\rightarrow} R^{\bullet} \stackrel{\sim}{\rightarrow} c\left(\Delta^{1}\right)$ be a factorization into a Reedy acyclic cofibration followed by Reedy fibration (which will necessarily be acyclic as well). By [12, Proposition 15.3.11] the maps $R^{n} \rightarrow \Delta^{1}$ are Joyal fibrations. Since $\Delta^{1}$ is Joyal fibrant (being the nerve of a category), the objects $R^{n}$ are fibrant as well.

For a simplicial set $X$, we denote by $\Delta X$ the category of simplices of $X$.

5.2 Proposition If $S$ is fibrant in $s \mathscr{Y e t}_{J}$ and $a, b \in S_{0}$, then there is a natural commutative diagram in which all the maps are Kan equivalences:

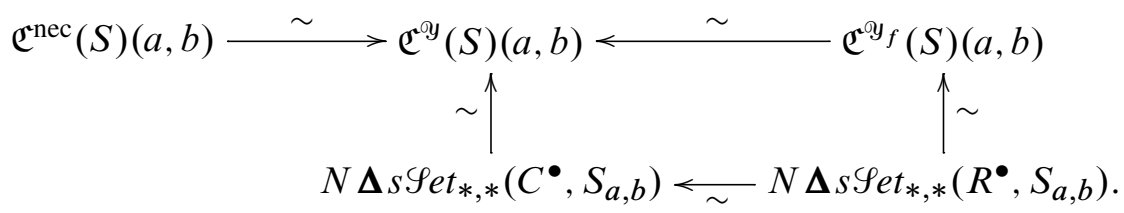


Proof The map $\mathfrak{C}^{\text {nec }}(S)(a, b) \rightarrow \mathfrak{C}^{0 y}(S)(a, b)$ is induced by the inclusion of categories $\mathcal{N e c} \hookrightarrow \mathscr{Y}$, and [8, Proposition 5.6] shows that is a Kan equivalence. The map $\mathfrak{C}^{y_{f}}(S)(a, b) \rightarrow \mathfrak{C}^{a y}(S)(a, b)$ is the nerve of the evident inclusion of overcategories $j:\left(\mathscr{Y}_{f} \downarrow S_{a, b}\right) \rightarrow\left(\mathscr{Y}_{\downarrow} \downarrow S_{a, b}\right)$. Let us show it is a Kan equivalence. For a simplicial set $X$, let $X \stackrel{\sim}{\hookrightarrow} \hat{X}$ denote a (functorial) fibrant replacement of $X$ in $s$ Set $_{J}$. Since $S$ is fibrant, there is a map $\widehat{S} \rightarrow S$ such that the composition $S \rightarrow \widehat{S} \rightarrow S$ is the identity. Define a functor

$$
F:\left(\mathscr{Y} \downarrow S_{a, b}\right) \rightarrow\left(\mathscr{Y}_{f} \downarrow S_{a, b}\right)
$$

by sending the pair $[Y, Y \rightarrow S]$ to the pair $[\hat{Y}, \hat{Y} \rightarrow \widehat{S} \rightarrow S]$. For this to make sense we need to know that $\hat{Y}$ is in $\mathscr{Y}_{f}$; this is true because changing from $Y \rightarrow \widehat{Y}$ does not change the Dwyer-Kan mapping space $\operatorname{hMap}(-)(a, b)$ nor, by [8, Proposition 6.6], the $\mathfrak{C}(-)(a, b)$ mapping space. It is easy to see that there is a natural transformation between the composite $j F$ (resp. $F j$ ) and the identity, so $j$ induces a Kan equivalence of the nerves.

Next consider the map $N \Delta s \mathscr{S e t}_{*, *}\left(R^{\bullet}, S_{a, b}\right) \rightarrow \mathfrak{C}^{\mathrm{y}_{f}}(S)(a, b)$. This is again the nerve of a functor

$$
f: \Delta s \mathscr{S e t}_{*, *}\left(R^{\bullet}, S_{a, b}\right) \rightarrow\left(\mathscr{Y}_{f} \downarrow S_{a, b}\right)
$$

which sends [[n], $R^{n} \rightarrow S$ ] to $\left[R^{n}, R^{n} \rightarrow S\right]$. We will verify that the overcategories of $f$ are contractible, hence $f$ induces a Kan equivalence of the nerves by Quillen's Theorem A. (For typographical reasons, we will drop the subscripts $a, b$, etc.) Pick an object $y=[Y, Y \rightarrow S]$ in $\left(y_{f} \downarrow S\right)$. The overcategory $(f \downarrow y)$ has objects $\left[[n], R^{n} \rightarrow Y\right]$ and the evident morphisms; that is, $(f \downarrow y)=\Delta s \operatorname{Set}_{*, *}\left(R^{\bullet}, Y\right)$. But since $Y$ is Joyal fibrant, $s \mathscr{S e t}_{*, *}\left(R^{\bullet}, Y\right)$ is a model for $\operatorname{hMap}(Y)(a, b)$, and this is contractible because $Y \in \mathscr{Y}_{f}$.

The map $s \mathscr{S e t}_{*, *}\left(R^{\bullet}, S\right) \rightarrow s \mathscr{S e t}_{*, *}\left(C^{\bullet}, S\right)$ is a Kan equivalence because $C^{\bullet} \rightarrow R^{\bullet}$ is a Reedy weak equivalence between Reedy cofibrant objects and $S$ is Joyal fibrant; see $[12,16.5 .5]$. Hence, the map $N \Delta s \mathscr{S e t}_{*, *}\left(R^{\bullet}, S_{a, b}\right) \rightarrow N \Delta s \operatorname{Set}_{*, *}\left(C^{\bullet}, S_{a, b}\right)$ is a Kan equivalence, using the fact that $N \Delta K \simeq K$, for any simplicial set $K$ (see [12, Theorem 18.9.3]).

The final map $N \Delta s \mathscr{S e t}_{*, *}\left(C^{\bullet}, S_{a, b}\right) \rightarrow \mathfrak{C}^{\mathrm{gy}}(S)(a, b)$ is a Kan equivalence by the two-out-of-three property.

5.3 Corollary Let $C^{\bullet}$ be any cosimplicial resolution for $\Delta^{1}$ in $s \mathscr{S e t}_{*, *}$. For a quasicategory $S$ and $a, b \in S_{0}$, there is a natural zigzag of Kan equivalences between $\mathfrak{C}(S)(a, b)$ and $\operatorname{hMap}(S)(a, b)={ }_{s} \mathscr{S e t}_{*, *}\left(C^{\bullet}, S_{a, b}\right)$. 
Proof Recall from [8, Theorem 5.3] that there is a natural zigzag of Kan equivalences between $\mathfrak{C}^{\text {nec }}(S)(a, b)$ and $\mathfrak{C}(S)(a, b)$. Also recall that for any simplicial set $K$, there is a natural zigzag of Kan equivalences between $K$ and $N \Delta K$ by [12, Theorem 18.9.3]. The corollary follows immediately from combining these facts with Proposition 5.2.

For the rest of this section we write $\mathscr{A}=s \mathscr{S e t}_{*, *}$, to ease the typography. By "simple zigzag" we will mean a diagram of the form $A \rightarrow B \leftarrow C$ or $A \leftarrow B \rightarrow C$.

Proposition 5.2 gives a simple zigzag of Kan equivalences between $\mathfrak{C}^{\text {nec }}(S)(a, b)$ and $N \boldsymbol{\Delta} s \mathscr{S e t}_{*, *}\left(C^{\bullet}, S_{a, b}\right)$ for any cosimplicial resolution $C^{\bullet}$ of $\Delta^{1}$ in $s \mathscr{S e t}_{J}$. In Proposition 5.6 we will present another simple zigzag which is sometimes useful. Define

$$
\phi:\left(\mathcal{N e c} \downarrow S_{a, b}\right) \rightarrow \mathscr{A} W^{-1} \mathscr{A}\left(\Delta^{1}, S_{a, b}\right)
$$

by sending $[T, T \rightarrow S]$ to $\left[\Delta^{1} \rightarrow \Delta[T] \leftarrow T \rightarrow S\right]$ (for the notation $\mathscr{A} W^{-1} \mathscr{A}$, see Section 3.2). Here $\Delta[T]$ is the associated simplex to $T$, described in [8, Section 3], which is functorial in $T$. The map $\Delta^{1} \rightarrow \Delta[T]$ is the unique 1 -simplex connecting the initial and final vertices. Note that there is also a functor

$$
j: \Delta s \mathscr{S e t}_{*, *}\left(C^{\bullet}, S\right) \rightarrow \mathscr{A} W^{-1} \mathscr{A}\left(\Delta^{1}, S\right)
$$

which sends $\left[[n], C^{n} \rightarrow S\right]$ to $\left[\Delta^{1} \stackrel{\text { id }}{\rightarrow} \Delta^{1} \stackrel{\leftarrow}{\leftarrow} C^{n} \rightarrow S\right]$, and by Remark 3.5 this functor induces a Kan equivalence on nerves.

5.6 Proposition For any fibrant simplicial set $S \in s \mathscr{S}_{e} t_{J}$ and $a, b \in S_{0}$, the maps

$$
\mathfrak{C}^{\mathrm{nec}}(S)(a, b) \stackrel{N \phi}{\longrightarrow} N\left[\mathscr{A} W^{-1} \mathscr{A}\left(\Delta^{1}, S_{a, b}\right)\right] \stackrel{N j}{\longleftarrow} N\left[\Delta s \operatorname{Set}_{*, *}\left(C^{\bullet}, S_{a, b}\right)\right],
$$

are Kan equivalences, where $\phi$ and $j$ are as in (5.4) and (5.5).

Proof We consider the following diagram of categories, where we have suppressed all mention of $a$ and $b$, but everything is suitably over $\partial \Delta^{1}$ :

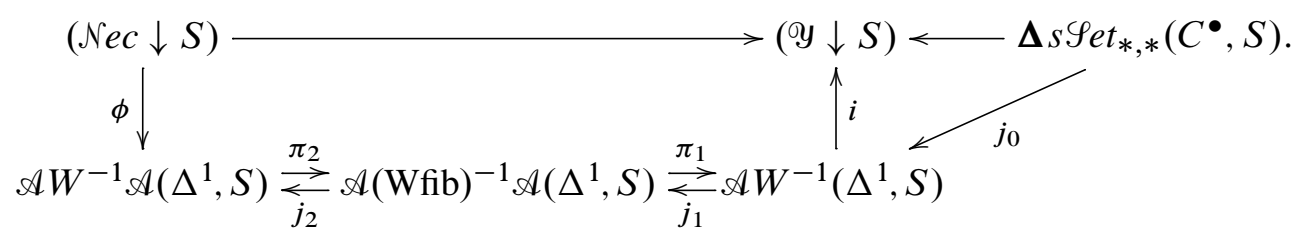

The nerve of each map in the top row is from Proposition 5.2, where it is shown to be a Kan equivalence. The map $\phi$ was defined above. We describe the maps $j_{0}, j_{1}, j_{2}, i, \pi_{1}$, and $\pi_{2}$ now (in that order). The symbol " $\sim$ " in this proof always denotes a Joyal equivalence. 
The map $j_{0}$ sends $\left[[n], C^{n} \rightarrow S\right]$ to $\left[\Delta^{1} \stackrel{\sim}{\leftarrow} C^{n} \rightarrow S\right] ; j_{1}$ sends $\left[\Delta^{1} \leftarrow X \rightarrow S\right]$ to $\left[\Delta^{1} \stackrel{\text { id }}{\rightarrow} \Delta^{1} \stackrel{\sim}{\leftarrow} X^{\prime} \rightarrow S\right]$, where $X \stackrel{\sim}{\lessgtr} X^{\prime} \rightarrow \Delta^{1} \times S$ is a functorial factorization of $X \rightarrow \Delta^{1} \times S$; and $j_{2}$ is induced by the inclusion Wfib $\hookrightarrow W$. Note that there is a natural transformation $j \rightarrow j_{2} j_{1} j_{0}$, so these maps induce homotopic maps on nerves. The map $i$ sends $\left[\Delta^{1} \leftarrow X \rightarrow S\right]$ to the pair $[X, X \rightarrow S]$ (note that if $X \simeq \Delta^{1}$ then $X \in \mathcal{Y}$ by [8, Proposition 6.6] and the homotopy invariance of the Dwyer-Kan mapping spaces). Finally, the maps $\pi_{1}$ and $\pi_{2}$ are functors giving homotopy inverses to $j_{1}$ and $j_{2}$, as in the proof of Proposition 3.3. The functor $\pi_{1}$ sends $\left[\Delta^{1} \rightarrow X \stackrel{\leftarrow}{\leftarrow} \rightarrow S\right]$ to $\left[\Delta^{1} \leftarrow\left(\Delta^{1} \times_{X} Y\right) \rightarrow S\right]$, and $\pi_{2}$ sends the zigzag $\left[\Delta^{1} \rightarrow X \leftarrow Y \rightarrow S\right.$ ] to $\left[\Delta^{1} \rightarrow X \stackrel{\sim}{\leftarrow} Y^{\prime} \rightarrow S\right]$ where $Y^{\prime}$ is obtained from the functorial factorization of $Y \rightarrow X \times S$ into $Y \stackrel{\sim}{\rightarrow} Y^{\prime} \rightarrow X \times S$. It is easy to see that there are natural transformations between the composites $j_{i} \pi_{i}, \pi_{i} j_{i}$, and their respective identities, thus showing that these maps are homotopy inverses.

Next one should check that the functor $i \pi_{1} \pi_{2} \phi$ is connected to the top map (Nec $\left.\downarrow S\right) \rightarrow$ (Y $\downarrow S$ ) by a zigzag of natural transformations (this is easy), and hence the two maps induce homotopic maps on nerves. So the (nerve of the) large rectangle in the above diagram commutes in the homotopy category. The right-hand triangle commutes on the nose.

The map $j_{0}$ induces a Kan equivalence on nerves by Remark 3.5. Returning to our original diagram and the sentence immediately following it, the two-out-of-three property implies that $i$ induces a Kan equivalence on nerves. We have already shown that $\pi_{1} \pi_{2}$ and $j_{2} j_{1}$ do so as well; therefore the same is true for $\phi$ and $j$.

5.7 Remark The above result in some sense explains why necklaces might arise in models for mapping spaces, as they did in [8]. If $T$ is a necklace then a map $T \rightarrow S_{a, b}$ gives us, in a canonical way, a zigzag

$$
\Delta^{1} \hookrightarrow \Delta[T] \stackrel{\sim}{\longleftarrow} T \rightarrow S
$$

in $\mathscr{A} W^{-1} \mathscr{A}\left(\Delta^{1}, S\right)$, which represents a map $\Delta^{1} \rightarrow S$ in $\operatorname{Ho}\left(s \mathscr{S e t}_{J}\right)$.

\subsection{The counit of rigidification}

Our next result concerns the counit $\epsilon: \mathfrak{C} N \rightarrow \operatorname{id}_{s \varphi_{a t}}$ for the adjunction $\mathfrak{C}: s \mathscr{S e t}_{J} \rightleftarrows$ $s$ bat: $N$. The proof involves only a slight enhancement of Proposition 5.2. For a proof using very different methods, see [16, Theorem 2.2.0.1].

Recall that a simplicial category is called fibrant [2] if all its mapping spaces are Kan complexes. 
5.9 Proposition Let $\mathscr{D}$ be a fibrant simplicial category. Then the counit map $\mathfrak{C} N \mathscr{D} \rightarrow \mathscr{D}$ is a weak equivalence in $s^{C}$ at .

Proof Since $\mathfrak{C}(N \mathscr{D})$ is a simplicial category with the same object set as $\mathscr{D}$, it suffices to show that for any $a, b \in \mathrm{ob} \mathscr{D}$ the map

$$
\mathfrak{C}(N \mathscr{D})(a, b) \rightarrow \mathscr{D}(a, b)
$$

is a Kan equivalence.

Let $C^{\bullet}$ be the cosimplicial resolution $C_{R}^{\bullet}$ from Section 4, so that we have $C^{n}=$ $\left(\Delta^{n} \star \Delta^{0}\right) / \Delta^{n}$. Observe that $\mathfrak{C}\left(C^{n}\right)$ is a simplicial category with two objects 0 and 1 , and following [16, Section 2.2.2] let $Q^{n}$ denote the mapping space $\mathfrak{C}\left(C^{n}\right)(0,1)$. By [8, Proposition 6.6] and Proposition 4.5(c) the map $Q_{n} \rightarrow \mathfrak{C}\left(\Delta^{1}\right)(0,1)=*$ is a Kan equivalence, hence $Q^{n}$ is contractible. Also, since $C_{R}^{\bullet}$ is Reedy cofibrant it follows readily that $Q^{\bullet}$ is also Reedy cofibrant. So the cosimplicial space $Q^{\bullet}$ is a cosimplicial resolution of a point in $s \mathscr{S e t}_{K}$.

Consider the following diagram in $s \mathscr{S e t}_{K}$ :

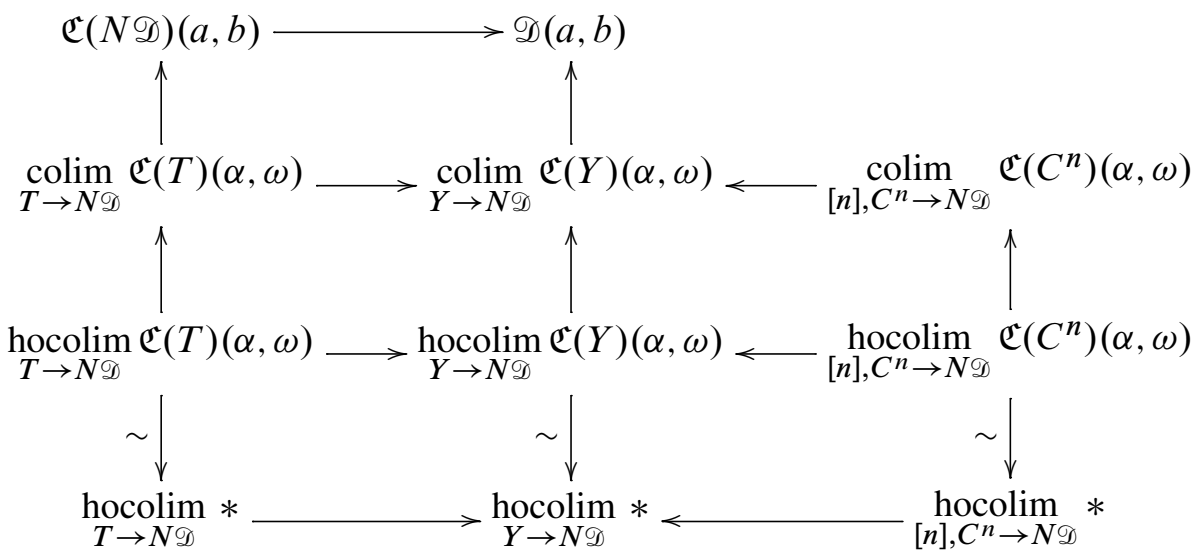

For the colimits in the left-hand column the indexing category is $\left(\mathcal{N e c} \downarrow N \mathscr{D}_{a, b}\right)$. For the middle column it is ( $\mathscr{\downarrow} \downarrow N \mathscr{D}_{a, b}$ ), where $Y$ is the category of gadgets described at the beginning of this section. For the right-hand column the colimits are indexed by the category $\Delta s \mathscr{S e t}_{*, *}\left(C^{\bullet}, N \mathscr{D}_{a, b}\right)$. The maps between columns (except at the very top) come from the evident maps between indexing categories. Finally, the top vertical map in the middle column is applied to $Y \rightarrow N \mathscr{D}$ by taking its adjoint to give $\mathfrak{C}(Y) \rightarrow \mathscr{D}$, and then using the induced map $\mathfrak{C}(Y)(\alpha, \omega) \rightarrow \mathscr{D}(a, b)$. It is easy to see that the diagram commutes. 
The indicated maps are Kan equivalences because the mapping spaces in $\mathfrak{C}(T), \mathfrak{C}(Y)$ and $\mathfrak{C}\left(C^{n}\right)$ are all contractible. The bottom horizontal row is

$$
\mathfrak{C}^{\text {nec }}(N \mathscr{D})(a, b) \rightarrow \mathfrak{C}^{\mathfrak{y}}(N \mathscr{D})(a, b) \leftarrow N \Delta s \mathscr{S e t}_{*, *}\left(C^{\bullet}, N \mathscr{D}_{a, b}\right)
$$

by [8, Section 5], and these maps are Kan equivalences by Proposition 5.2. It follows that the horizontal maps in the third row are all Kan equivalences as well.

Now consider the composite

$$
\underset{[n], \mathfrak{C}\left(C^{n}\right) \rightarrow \mathscr{D}}{\operatorname{hocolim}} \mathfrak{C}\left(C^{n}\right)(\alpha, \omega) \rightarrow \underset{[n], \mathfrak{C}\left(C^{n}\right) \rightarrow \mathscr{D}}{\operatorname{colim}} \mathfrak{C}\left(C^{n}\right)(\alpha, \omega) \rightarrow \mathscr{D}(a, b) .
$$

To give a map $\mathfrak{C}\left(C^{n}\right) \rightarrow \mathscr{D}$ over $a, b$ is exactly the same as giving a map $Q^{n}=$ $\mathfrak{C}\left(C^{n}\right)(\alpha, \omega) \rightarrow \mathscr{D}(a, b)$. So the above maps may also be written as

$$
\underset{[n], Q^{n} \rightarrow \mathscr{D}(a, b)}{\operatorname{hocolim}} Q^{n} \longrightarrow \underset{[n], Q^{n} \rightarrow \mathscr{D}(a, b)}{\operatorname{colim}} Q^{n} \longrightarrow \mathscr{D}(a, b) .
$$

By Lemma 5.10 below (using that $\mathscr{D}(a, b)$ is a Kan complex), this composite is a Kan equivalence.

It now follows from our big diagram that $\operatorname{hocolim}_{Y \rightarrow N \mathscr{D}} \mathfrak{C}(Y)(\alpha, \omega) \rightarrow \mathscr{D}(a, b)$ is a Kan equivalence. Finally, by [8, Theorem 5.3] the map

$$
\underset{T \rightarrow N \mathscr{D}}{\operatorname{hocolim}} \mathfrak{C}(T)(\alpha, \omega) \rightarrow \mathfrak{C}(N \mathscr{D})(a, b)
$$

is a Kan equivalence (this is the map $\mathfrak{C}^{\text {hoc }}(N \mathscr{D})(a, b) \rightarrow \mathfrak{C}(N \mathscr{D})(a, b)$ from the statement of that theorem). It now follows at once that $\mathfrak{C}(N \mathscr{D})(a, b) \rightarrow \mathscr{D}(a, b)$ is a Kan equivalence.

5.10 Lemma Let $U^{\bullet}$ be any cosimplicial resolution of a point with respect to $s \operatorname{Set}_{K}$. Then for any Kan complex $X$, the composite

$$
\operatorname{hocolim}_{[n], U^{n} \rightarrow X} U^{n} \longrightarrow \operatorname{colim}_{[n], U^{n} \rightarrow X} U^{n} \longrightarrow X .
$$

is a Kan equivalence.

Proof The result is true for the cosimplicial resolution $\Delta^{\bullet}$ by a standard result; see [7, Proposition 19.4], for instance. There is a zigzag $U^{\bullet} \rightarrow V^{\bullet} \leftarrow \Delta^{\bullet}$ of Reedy weak equivalences, where $V^{\bullet}$ is a cofibrant-fibrant replacement of $\Delta^{\bullet}$ in $c\left(s \operatorname{Set}_{K}\right)$. Because of this it is sufficient to show that if $U^{\bullet} \rightarrow V^{\bullet}$ is a map between cosimplicial resolutions of a point and we know the result for one of them, then we also know it for the other. 
Write $M$ to denote ${ }_{s} \mathscr{S e t}_{K}$. Let $I=\Delta M\left(U^{\bullet}, X\right)$ and $J=\Delta M\left(V^{\bullet}, X\right)$, and observe that our map $U^{\bullet} \rightarrow V^{\bullet}$ induces a functor $f: J \rightarrow I$.

Let $\Gamma_{U}: I \rightarrow M$ be the functor $\left[[n], U^{n} \rightarrow X\right] \mapsto U^{n}$ and let $\Gamma_{V}: J \rightarrow M$ be the functor $\left[[n], V^{n} \rightarrow X\right] \mapsto V^{n}$. Finally, let $\Theta: J \rightarrow M$ be the functor $\left[[n], V^{n} \mapsto X\right] \mapsto U^{n}$. Note that there is a natural transformation $\Theta \rightarrow \Gamma_{V}$, and also that $\Theta=\Gamma_{U} \circ f$.

One considers the following diagram:

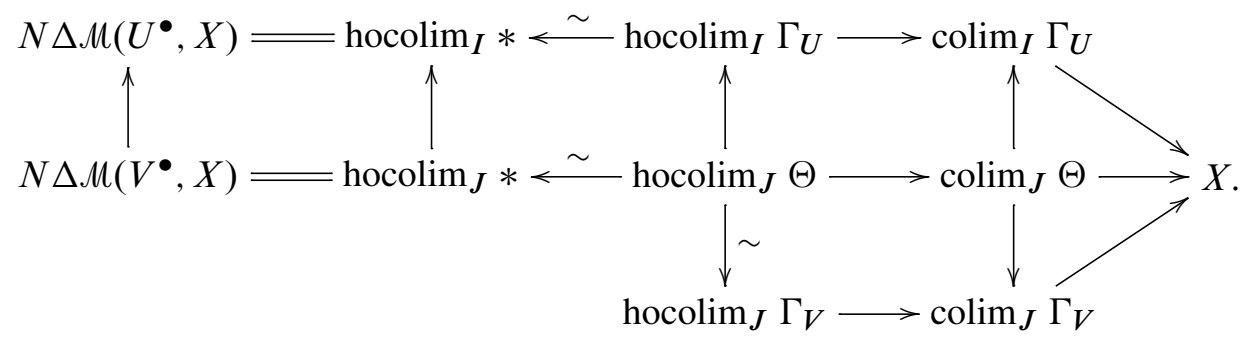

The maps labelled $\sim$ are Kan equivalences because all the values of $\Gamma_{V}, \Gamma_{U}$, and $\Theta$ are contractible.

The key observation is that the map $M\left(V^{\bullet}, X\right) \rightarrow M\left(U^{\bullet}, X\right)$ is a Kan equivalence by $[12,16.5 .5]$, since both $V^{\bullet}$ and $U^{\bullet}$ are cosimplicial resolutions of a point in ${ }_{s} \mathscr{S e t}_{K}$ and $X$ is Kan fibrant. It follows that $N \Delta M\left(V^{\bullet}, X\right) \rightarrow N \Delta M\left(U^{\bullet}, X\right)$ is also a Kan equivalence, and applying the two-out-of-three axiom to the diagram we obtain that $\operatorname{hocolim}_{I} \Gamma_{U} \rightarrow X$ is a Kan equivalence if and only if $\operatorname{hocolim}_{J} \Gamma_{V} \rightarrow X$ is a Kan equivalence. This is what we wanted.

\section{Relative mapping spaces}

In previous sections we studied the mapping spaces $\operatorname{hMap}_{\left(\partial \Delta^{1} \downarrow_{s} \varphi_{e t_{J}}\right)}\left(\Delta^{1}, X\right)$. There is an evident generalization of this construction which replaces $\partial \Delta^{1} \longrightarrow \Delta^{1}$ with an arbitrary cofibration $A \succ B$. This turns out to be useful in Section 7 and Section 8 , and potentially in outside applications. The point can immediately be seen in Proposition 6.3 below: even if one is mainly interested in the case where $A \succ B$ is $\partial \Delta^{1} \succ \Delta^{1}$, adjointness arguments can quickly take one into a more general setting. The purpose of the present section is to develop the basic properties of these relative mapping spaces.

Recall from Lemma 4.2 that the map $A \times E^{n} \rightarrow A$ is a Joyal acyclic fibration, for all $A$. It follows that the cosimplicial object $[n] \mapsto A \times E^{n}$ is a cosimplicial resolution for $A$ with respect to $s \mathscr{S e t}_{J}$. 
6.1 Definition Fix a cofibration $A \succ B$.

(a) Define $C_{E}^{\bullet}(B, A)$ to be the cosimplicial object obtained as the pushout

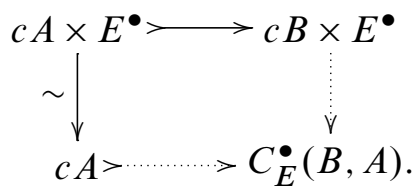

Note that the pushout of a Joyal equivalence is still a Joyal equivalence by left properness, and so $C_{E}^{\bullet}(B, A)$ is a cosimplicial resolution of $B$ in the model category $\left(A \downarrow s \mathscr{S e t}_{J}\right)$.

(b) For any quasi-category $X$ and any fixed map $f: A \rightarrow X$, let $\operatorname{hMap}_{A}(B, X)$ be the pullback of simplicial sets

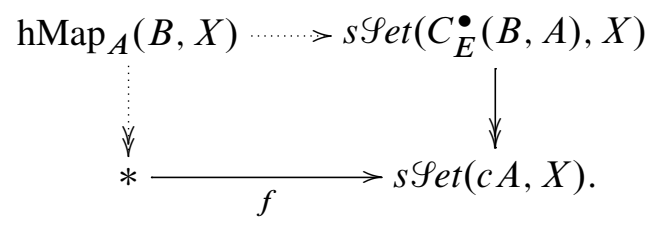

Note that $\operatorname{set}(c A, X)$ is a discrete simplicial set, and also that the right vertical map is a Kan fibration since $c A \rightarrow C_{E}^{\bullet}(B, A)$ is a Reedy cofibration. One should also observe that $\operatorname{hMap}_{A}(B, X)$ depends on the map $f$ as well as on the fixed cofibration, although this is obscured in the notation.

6.2 Remark The simplicial set $\operatorname{hMap}_{A}(B, X)$ is simply a particular model for the homotopy function complex from $B$ to $X$ in the model category $\left(A \downarrow s \mathscr{S e t}_{J}\right)$. Note that of the four resolutions considered in Proposition 4.5, only the $E^{\bullet}$ resolution is relevant in our present context; the others are specific to $\partial \Delta^{1} \rightarrow \Delta^{1}$.

For later use, observe that $\operatorname{hMap}_{A}(B, X)$ can also be described as the pullback

$$
* \longrightarrow s \operatorname{Set}\left(A \times E^{\bullet}, X\right) \longleftarrow s \operatorname{Set}\left(B \times E^{\bullet}, X\right)
$$

where the left map is the composite $* \rightarrow s \operatorname{Set}(c A, X) \rightarrow s \operatorname{Set}\left(A \times E^{\bullet}, X\right)$.

6.3 Proposition Let $A \succ B$ be a cofibration, and let $C$ be any simplicial set. Let $X$ be a quasi-category and $f: A \rightarrow X^{C}$ be a map. Then

$$
\operatorname{hMap}_{A}\left(B, X^{C}\right) \cong \operatorname{hMap}_{A \times C}(B \times C, X)
$$

where the right mapping space is relative to the map $A \times C \rightarrow X$ adjoint to $f$.

Proof Easy, using the second statement in Remark 6.2. 
Given a square

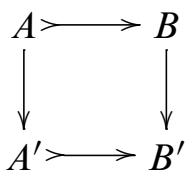

and a map $A^{\prime} \rightarrow X$, there is an induced map $\operatorname{hMap}_{A^{\prime}}\left(B^{\prime}, X\right) \rightarrow \operatorname{hMap}_{A}(B, X)$. The next two results give properties of these natural maps.

6.5 Proposition Assume given a square such as (6.4), and let $L: A^{\prime} \amalg_{A} B \rightarrow B^{\prime}$ denote the induced map. Then for any quasi-category $X$ and any map $f: A^{\prime} \rightarrow X$, the induced map $\operatorname{hMap}_{A^{\prime}}\left(B^{\prime}, X\right) \rightarrow \operatorname{hMap}_{A}(B, X)$ is a Kan fibration if $L$ is a cofibration, and it is a Kan acyclic fibration if $L$ is a Joyal acyclic cofibration.

\subsection{Lemma Let}

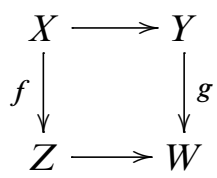

be a diagram of simplicial sets, and let $z \in Z$ be some basepoint. Let $F_{1}$ be the fiber of $f$ over $z$, and let $F_{2}$ be the fiber of $g$ over the image of $z$. Then if the "matching map" $X \rightarrow Y \times{ }_{Z} W$ has the right-lifting-property with respect to some map $A \rightarrow B$, so does the map of fibers $F_{1} \rightarrow F_{2}$.

In particular, if the matching map is a Kan fibration (resp. acyclic fibration) so is $F_{1} \rightarrow F_{2}$.

Proof Left to the reader.

Proof Consider the diagram below:

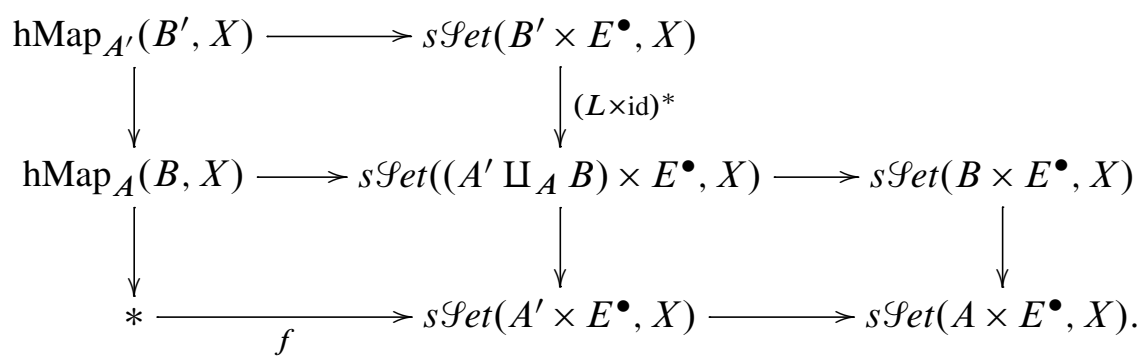

Note that the two large rectangles are pullback squares, and that the lower right square is also a pullback. It follows by standard results about pullback squares in any category that the lower left square is also a pullback, and then that the same is true for the upper 
left square. The desired result now follows directly, since the map labelled $(L \times \mathrm{id})^{*}$ will be either a Kan fibration or acyclic Kan fibration under the respective hypothesis on $L$.

6.7 Proposition Let $A \succ B$ be a cofibration, and let $B_{1} \succ B$ and $B_{2} \succ B$ be such that $B_{1} \cup B_{2}=B$. Let $X$ be a quasi-category and $f: A \rightarrow X$ any map. Then the following is a pullback square, as well as a homotopy pullback square in $s \mathscr{S e t}_{K}$ :

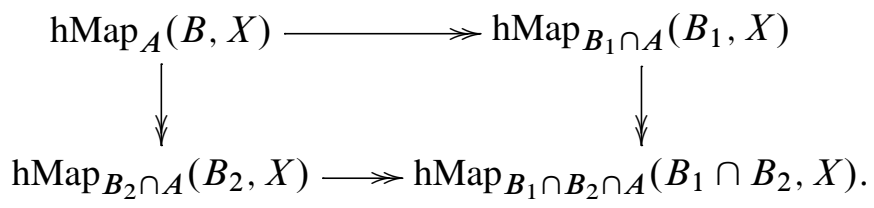

Proof Note that the pullback statement immediately implies the homotopy pullback statement, since we know by four applications of Proposition 6.5 that the indicated maps are Kan fibrations.

To prove the pullback statement we simply consider the following diagram:

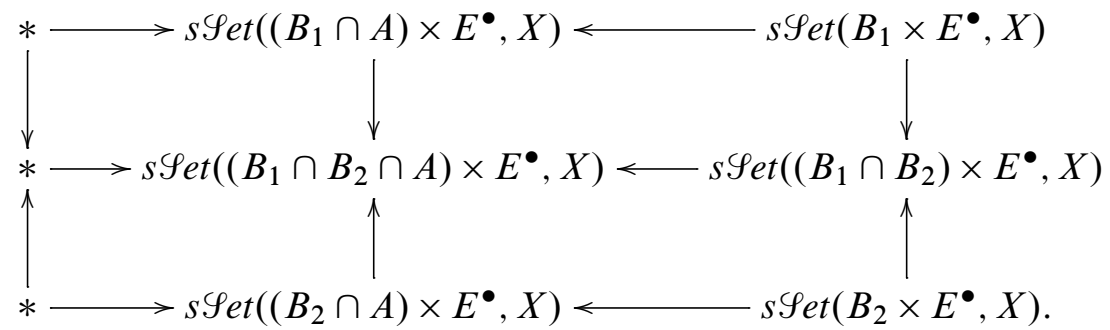

The limit of this diagram may be constructed by first forming the pullbacks of the columns, and then forming the resulting pullback; and it may also be constructed by first forming the pullbacks of the rows and then forming the resulting pullback. The former method gives the pullback of

$$
* \rightarrow s \operatorname{Set}\left(A \times E^{\bullet}, X\right) \leftarrow s \mathscr{S e t}\left(B \times E^{\bullet}, X\right),
$$

which is just $\operatorname{hMap}_{A}(B, X)$. The latter method gives the pullback of

$$
\operatorname{hMap}_{B_{1} \cap A}\left(B_{1}, X\right) \rightarrow \operatorname{hMap}_{B_{1} \cap B_{2} \cap A}\left(B_{1} \cap B_{2}, X\right) \leftarrow \operatorname{hMap}_{B_{2} \cap A}\left(B_{2}, X\right) .
$$

This completes the proof.

The following proposition demonstrates the use of the above results. Morally, these results allow the analysis of any relative mapping space to be reduced to that of mapping spaces relative to $\partial \Delta^{1} \succ \Delta^{1}$. 
6.8 Proposition Suppose that $X \rightarrow Y$ is a map of quasi-categories and that for all $a, b \in X$ the induced map $\operatorname{hMap}_{\partial \Delta^{1}}\left(\Delta^{1}, X\right) \rightarrow \operatorname{hMap}_{\partial \Delta^{1}}\left(\Delta^{1}, Y\right)$ is a Kan equivalence. Then for any pair of $1-$ simplices $f, g: \Delta^{1} \rightarrow X$ (regarded as a single map $\partial \Delta^{1} \times \Delta^{1} \rightarrow X$ ), the map

$$
\operatorname{hMap}_{\partial \Delta^{1} \times \Delta^{1}}\left(\Delta^{1} \times \Delta^{1}, X\right) \rightarrow \operatorname{hMap}_{\partial \Delta^{1} \times \Delta^{1}}\left(\Delta^{1} \times \Delta^{1}, Y\right)
$$

is also a Kan equivalence.

Proof Let $S$ and $T$ be the nondegenerate 2 -simplices [00, 01, 11] and [00, 10, 11] in $\Delta^{1} \times \Delta^{1}$. Write $S_{0}=[00,01] \cup[11]$ and $T_{0}=[00] \cup[10,11]$. Then by Proposition 6.7 the map in which we are interested is the induced map on pullbacks of the following diagram:

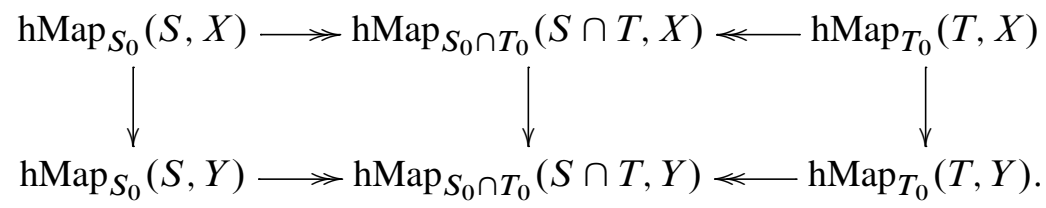

Now, $S_{0} \cap T_{0} \hookrightarrow S \cap T$ is the inclusion of the boundary of a 1-simplex; hence the assumptions of the proposition imply that the middle vertical map is a Kan equivalence. To analyze the left vertical map in (6.9) we let $S_{1}=[01,11]$ and consider the square

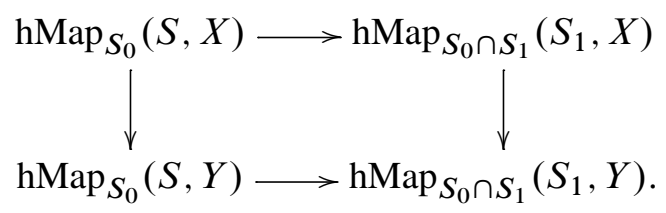

As the map $S_{1} \amalg_{S_{1} \cap S_{0}} S_{0} \hookrightarrow S$ is a Joyal acyclic cofibration, it follows by Proposition 6.5 that the two horizontal maps are Kan equivalences. Finally, since $S_{0} \cap S_{1} \hookrightarrow S_{1}$ is the inclusion of the boundary of a 1-simplex, the right vertical map is a Kan equivalence by assumption. Therefore the left vertical map is a Kan equivalence as well.

A similar proof shows that the right vertical map in (6.9) is a Kan equivalence, which means this is true for all the vertical maps. Since the horizontal maps are Kan fibrations, it follows that the induced map on pullbacks is a Kan equivalence as well. This is what we wanted.

\section{DK-equivalences for quasi-categories}

In this section we introduce a new notion of equivalence for simplicial sets, called "DKequivalence." It is designed to be analogous to the corresponding notion for simplicial 
categories [2]. We will eventually prove that the class of DK-equivalences is the same as the class of Joyal equivalences. In the present section we set out to accomplish this by establishing some basic properties of DK-equivalences.

7.1 Definition A map $f: X \rightarrow Y$ of simplicial sets is said to be a $D K$-equivalence if two conditions are satisfied:

(1) The induced map $\operatorname{Ho}\left(s \operatorname{Set}_{J}\right)(*, X) \rightarrow \operatorname{Ho}\left(\operatorname{set}_{J}\right)(*, Y)$ is a bijection.

(2) For every two 0 -simplices $a, b \in X$, the induced map $\operatorname{hMap}_{s \varphi_{e t, *}}\left(\Delta^{1}, X\right) \rightarrow$ $\operatorname{hMap}_{s \varphi_{e t, *}}\left(\Delta^{1}, Y\right)$ is a Kan equivalence.

The following lemma gives us three ways of recognizing DK-equivalences:

7.2 Lemma Let $f: X \rightarrow Y$ be a Joyal fibration where both $X$ and $Y$ are quasicategories, and assume that $f$ satisfies condition (2) of Definition 7.1. Then the following statements are equivalent:

(a) $f$ has the right-lifting-property with respect to $\varnothing \rightarrow \Delta^{0}$ and $\{0,1\} \hookrightarrow E^{1}$.

(b) $[*, X]_{E^{1}} \rightarrow[*, Y]_{E^{1}}$ is a bijection.

(c) $f$ has the right-lifting-property with respect to $\varnothing \rightarrow \Delta^{0}$ (equivalently, $f$ is surjective).

(d) $\quad f$ satisfies condition (1) in Definition 7.1.

Proof Since $E^{1}$ is a cylinder object for $*$ in $s \mathscr{S e t}_{J}$, (b) and (d) are equivalent. We prove $(\mathrm{a}) \Rightarrow(\mathrm{b}) \Rightarrow(\mathrm{c}) \Rightarrow$ (a). The first implication is trivial, using the evident map of coequalizer diagrams defining $[-,-]_{E^{1}}$.

Assume (b) is true and pick a map $a: \Delta^{0} \rightarrow Y$. Since $[*, X]_{E^{1}} \rightarrow[*, Y]_{E^{1}}$ is surjective, there is a map $b: \Delta^{0} \rightarrow X$ and a map $h: E^{1} \rightarrow Y$ such that $h(0)=f(b)$ and $h(1)=a$. As $X \rightarrow Y$ is a Joyal fibration, it has the right-lifting-property with respect to $\{0\} \hookrightarrow E^{1}$. By lifting the evident square we find a preimage for $a$ in $X$.

Finally, assume (c) holds and suppose given a square

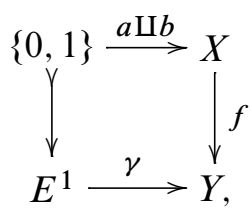


with $\gamma(0)=f(a)$ and $\gamma(1)=f(b)$. As $X \rightarrow Y$ has the right-lifting-property with respect to $\{0\} \rightarrow E^{1}$, we can use this to lift $\gamma$ to a map $\beta: E^{1} \rightarrow X$ satisfying $\beta(0)=a$. Setting $a^{\prime}=\beta(1)$, we then have $f(a)=f\left(a^{\prime}\right)=\gamma(1)$.

Let $F$ be the fiber of $f$ over $\gamma(1)$, and let $\partial \Delta^{1} \rightarrow F$ send $0 \mapsto a^{\prime}$ and $1 \mapsto b$. Then we obtain a pullback square

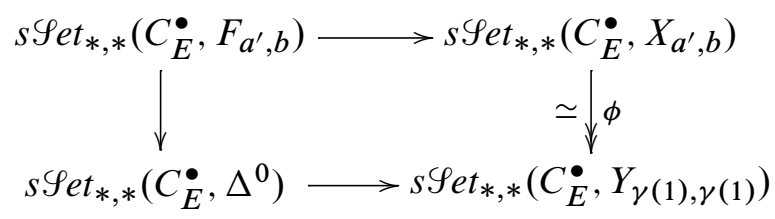

where $\phi$ is a Kan acyclic fibration by our assumptions on $f$. As the pullback of $\phi$ will also be a Kan acyclic fibration, this shows that $s \mathscr{S e t}_{*, *}\left(C_{E}^{\bullet}, F_{a^{\prime}, b}\right)$ is contractible. By Corollary 5.3 we then know that $\mathfrak{C}(F)\left(a^{\prime}, b\right)$ is contractible.

The same argument shows that $\mathfrak{C}(F)\left(a^{\prime}, a^{\prime}\right), \mathfrak{C}(F)(b, b)$, and $\mathfrak{C}(F)\left(b, a^{\prime}\right)$ are contractible, and this immediately yields that the objects $a^{\prime}$ and $b$ are isomorphic in the category $\pi_{0} \mathfrak{C}(F)$. Hence by Corollary 2.19 and Proposition 2.2 there is a map $E^{1} \rightarrow F$ connecting $a^{\prime}$ and $b$. Let $\delta$ denote the composite $E^{1} \rightarrow F \rightarrow X$.

Let $h$ be the composite

$$
E^{2} \stackrel{\pi}{\longrightarrow} E^{1} \stackrel{\gamma}{\longrightarrow} Y
$$

where $\pi$ sends $0 \mapsto 0,1 \mapsto 1$, and $2 \mapsto 1$. We then have a commutative square

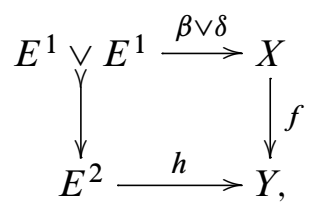

where the left vertical map is the evident inclusion. This map is a Joyal acyclic cofibration (both the domain and codomain are contractible in $s \mathscr{S e t}_{J}$ ), and so the above square has a lift $E^{2} \rightarrow X$. Precomposing this lift with the inclusion $E^{1} \hookrightarrow E^{2}$ sending $0 \mapsto 0$ and $1 \mapsto 2$, we obtain a lift of the original map $\gamma$.

7.3 Proposition Let $X, Y, X^{\prime}$, and $Y^{\prime}$ be quasi-categories.

(a) If $f: X \rightarrow Y$ is a Joyal fibration and a $D K$-equivalence, and $g: Y^{\prime} \rightarrow Y$ is any map, then the pullback $X \times_{Y} Y^{\prime} \rightarrow Y^{\prime}$ is a Joyal fibration and a $D K$-equivalence. 
(b) Let

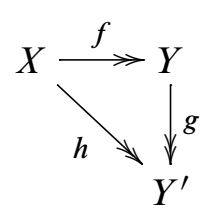

be a diagram in which all maps are Joyal fibrations. Then if two of the three maps are $D K$-equivalences, so is the third.

(c) Consider a diagram

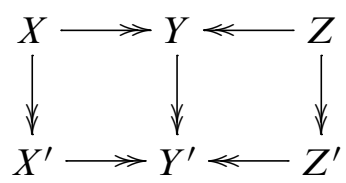

where all the maps are Joyal fibrations and the vertical maps are $D K$-equivalences. Let $P$ and $P^{\prime}$ be the pullbacks of the two rows, and assume that $P \rightarrow P^{\prime}$ is also a Joyal fibration. Finally, assume that $X \rightarrow X^{\prime} \times_{Y^{\prime}} Y$ and $Z \rightarrow Z^{\prime} \times_{Y^{\prime}} Y$ are Joyal fibrations. Then $P \rightarrow P^{\prime}$ is also a $D K$-equivalence.

Proof For (a), the pullback is certainly a Joyal fibration and its domain $X \times_{Y} Y^{\prime}$ is a quasi-category. Since the map $f$ has the RLP with respect to $\varnothing \rightarrow \Delta^{0}$, so does the pullback. So we need only check condition (2) of Definition 7.1. Let $a=\left(a_{1}, a_{2}\right)$ and $b=\left(b_{1}, b_{2}\right)$ be two points in $X \times_{Y} Y^{\prime}$, where $a_{1}, b_{1} \in X$ and $a_{2}, b_{2} \in Y^{\prime}$. Let $C^{\bullet}$ be a cosimplicial resolution for $\Delta^{1}$ in $\left(s \mathscr{S e t}_{*, *}\right)_{J}$. Then we have a pullback square

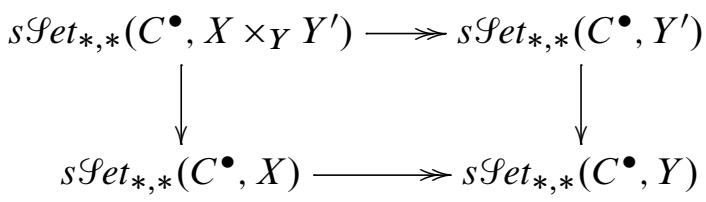

where the indicated maps are Kan fibrations. By assumption the bottom horizontal map is a Kan equivalence, hence so is the top horizontal map. This is what we wanted.

For (b), condition (1) of Definition 7.1 clearly satisfies the two-out-of-three property; we must check the same for condition (2). This is trivial in the case where $f$ and $g$ are DK-equivalences, and also in the case where $g$ and $h$ are DK-equivalences. Assume then that $f$ and $h$ are DK-equivalences. Given two points $a, b \in Y$, we may lift them to $a^{\prime}, b^{\prime} \in X$ because $f$ has the RLP with respect to $\varnothing \rightarrow \Delta^{0}$. At this point the proof becomes trivial again.

For (c), let $Q=X^{\prime} \times{ }_{Y^{\prime}} Y$ and $R=Z^{\prime} \times_{Y^{\prime}} Y$. The maps $Q \rightarrow X^{\prime}$ and $R \rightarrow Z^{\prime}$ are Joyal fibrations and DK-equivalences by part (a). Therefore the maps $X \rightarrow Q$ and 
$Z \rightarrow R$, which are Joyal fibrations by assumption, are also DK-equivalences by (b). In particular, these maps have the right-lifting-property with respect to $\varnothing \rightarrow \Delta^{0}$.

Let $a$ be a 0 -simplex in $P^{\prime}$. This gives rise to 0 -simplices $a_{1} \in X^{\prime}, a_{2} \in Y^{\prime}$, and $a_{3} \in Z^{\prime}$. Since $Y \rightarrow Y^{\prime}$ is a Joyal fibration and a DK-equivalence, there is a lift of $a_{2}$ to a point $b_{2}$ in $Y$. Then $\left(a_{1}, b_{2}\right)$ describes a 0 -simplex in $Q$, hence there is a point $b_{1}$ of $X$ lifting it. Likewise, $\left(a_{3}, b_{2}\right)$ describes a 0 -simplex of $R$, so there is a point $b_{3}$ in $Z$ lifting it. The pair $\left(b_{1}, b_{3}\right)$ gives a 0 -simplex of $P$ that lifts $a$. Hence $P \rightarrow P^{\prime}$ has the right-lifting-property with respect to $\varnothing \rightarrow \Delta^{0}$.

To see that $P \rightarrow P^{\prime}$ satisfies condition (2) of Definition 7.1, let $a$ and $b$ denote two points of $P$. Let $C^{\bullet}$ denote any cosimplicial resolution of $\Delta^{1}$ in $\left(s \mathscr{S e t}_{*, *}\right)_{J}$. Then we have a diagram

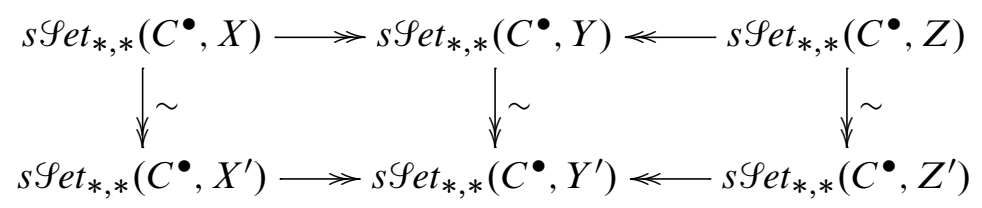

in which the indicated maps are Kan fibrations and Kan equivalences. By a standard property of simplicial sets, the induced map on pullbacks (from the top row to the bottom row) is a Kan equivalence. But the two pullbacks coincide with $s_{\operatorname{Set} t_{*}}\left(C^{\bullet}, P\right)$ and $s \mathscr{S e t}_{*, *}\left(C^{\bullet}, P^{\prime}\right)$, so we have shown that $\operatorname{hMap}_{s \mathscr{S e t}_{*, *}}\left(\Delta^{1}, P\right) \rightarrow \operatorname{hMap}_{s \mathscr{S e t}_{*, *}}\left(\Delta^{1}, P^{\prime}\right)$ is a Kan equivalence for every two points $a, b \in P$. This completes the proof.

\subsection{Comparing DK-equivalences and Joyal equivalences}

Clearly every Joyal equivalence is a DK-equivalence. In this section we will prove a partial converse, namely Proposition 7.6 below. The complete converse is proven in Proposition 8.1.

7.5 Lemma Let $X$ and $Y$ be quasi-categories and $f: X \rightarrow Y$ be a Joyal fibration and a $D K$-equivalence. Then for every $n \geq 0$ the following maps are also Joyal fibrations and $D K$-equivalences:
(a) $X^{\Delta^{n}} \rightarrow Y^{\Delta^{n}}$.
(b) $X^{\Lambda_{k}^{n}} \rightarrow Y^{\Lambda_{k}^{n}}$ for any $0<k<n$.
(c) $X^{\partial \Delta^{n}} \rightarrow Y^{\partial \Delta^{n}}$.
(d) $X^{\Delta^{n}} \rightarrow\left[Y^{\Delta^{n}} \times_{Y^{\partial \Delta^{n}}} X^{\partial \Delta^{n}}\right]$. 
Proof Note first that all the maps are Joyal fibrations between quasi-categories, by Proposition 2.15. We next prove that $X^{\Delta^{1}} \rightarrow Y^{\Delta^{1}}$ is a DK-equivalence. Condition (2) in the definition of DK-equivalence is verified by Proposition 6.8. Using Lemma 7.2, it will be enough to verify that $X^{\Delta^{1}} \rightarrow Y^{\Delta^{1}}$ is surjective. That is, we must show that $X \rightarrow Y$ has the right-lifting-property with respect to $\varnothing \rightarrow \Delta^{1}$.

Given a map $g: \Delta^{1} \rightarrow Y$, we may lift $g(0)$ and $g(1)$ to points $a$ and $b$ in $X$ since $X \rightarrow Y$ is surjective by Lemma 7.2. As the map $s \mathscr{S e t}_{*, *}\left(C_{E}^{\bullet}, X\right) \rightarrow s \mathscr{S e t}_{*, *}\left(C_{E}^{\bullet}, Y\right)$ is a Kan acyclic fibration, and $g$ represents a 0 -simplex in the target, we can lift $g$ to a 0 -simplex in the domain. This is what was wanted (recall that $C_{E}^{0}=\Delta^{1}$ ).

Now let $I[n]$ denote the spine $\operatorname{Spi}\left[\Delta^{n}\right]$, so that $I[n]$ consists of $n$ copies of $\Delta^{1}$ wedged together end-to-end. We next prove by induction that $X^{I[n]} \rightarrow Y^{I[n]}$ is a DK-equivalence for all $n \geq 1$. The case $n=1$ was handled above, so assume it is true for some $n \geq 1$. Note that $I[n+1]$ is a pushout of $I[n] \leftarrow \Delta^{0} \rightarrow \Delta^{1}$, and therefore $X^{I[n+1]}$ is the pullback of $X^{I[n]} \rightarrow X \leftarrow X^{\Delta^{1}}$. Consider the diagram

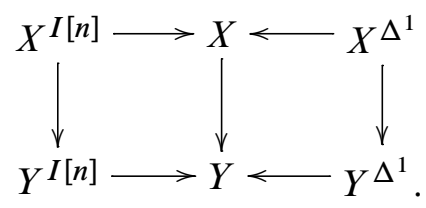

This diagram satisfies all the hypotheses of Proposition 7.3(c), so the induced map on pullbacks is a DK-equivalence. But this induced map is precisely $X^{I[n+1]} \rightarrow Y^{I[n+1]}$.

Now we turn to the proofs of (a) and (b). The inclusion $I[n] \hookrightarrow \Delta^{n}$ is a Joyal acyclic cofibration, so $X^{\Delta^{n}} \rightarrow X^{I[n]}$ is a Joyal acyclic fibration; in particular, it is a DKequivalence. Applying Proposition 7.3(b) (in various ways) to the diagram

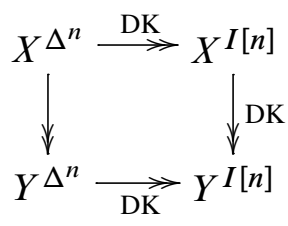

now gives that $X^{\Delta^{n}} \rightarrow Y^{\Delta^{n}}$ is a DK-equivalence.

For $0<k<n$, the inclusion $\Lambda_{k}^{n} \hookrightarrow \Delta^{n}$ is a Joyal acyclic cofibration, and therefore $X^{\Delta^{n}} \rightarrow X^{\Lambda_{k}^{n}}$ is a Joyal acyclic fibration. A similar argument to the last paragraph shows that $X^{\Lambda_{k}^{n}} \rightarrow Y^{\Lambda_{k}^{n}}$ is a DK-equivalence.

We will prove part (c) by induction. The cases $n=0$ and $n=1$ follow by hypothesis. For $n \geq 2$ note that $\partial \Delta^{n}$ is the pushout of $\Delta^{n-1} \leftarrow \partial \Delta^{n-1} \rightarrow \Lambda_{n-1}^{n}$. This leads us to 
the diagram

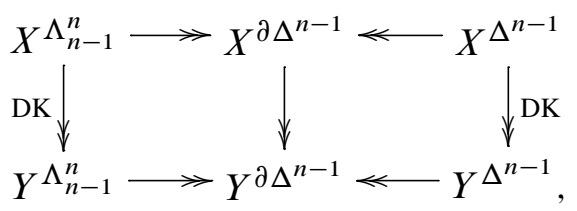

where the induced map on pullbacks is $X^{\partial \Delta^{n}} \rightarrow Y^{\partial \Delta^{n}}$. By parts (a) and (b) the indicated maps are DK-equivalences, and the middle map is a DK-equivalence by induction. One readily checks that the diagram satisfies the conditions of Proposition 7.3(c), hence $X^{\partial \Delta^{n}} \rightarrow Y^{\partial \Delta^{n}}$ is also a DK-equivalence.

Finally, to prove (d) let $n \geq 0$ and consider the diagram

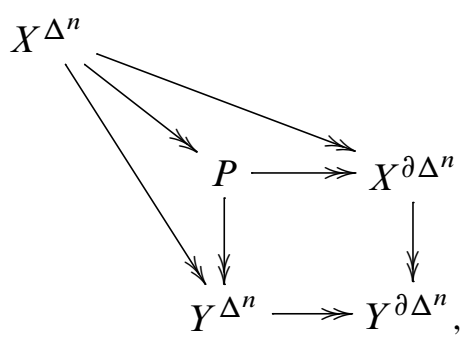

where $P$ is the pullback. We have proven that the right vertical map is a DKequivalence, hence so is the pullback $P \rightarrow Y^{\Delta^{n}}$ by Proposition 7.3(a). We have also proven that $X^{\Delta^{n}} \rightarrow Y^{\Delta^{n}}$ is a DK-equivalence, so the same is true for $X^{\Delta^{n}} \rightarrow P$ by Proposition 7.3(b).

7.6 Proposition If $X \rightarrow Y$ is a DK-equivalence between quasi-categories and a Joyal fibration then $X \rightarrow Y$ is a Kan acyclic fibration (and so, in particular, a Joyal equivalence).

Proof By Lemma 7.5(d) we know that for any $n \geq 0$ the map

$$
X^{\Delta^{n}} \rightarrow X^{\partial \Delta^{n}} \times_{Y^{\partial \Delta^{n}}} Y^{\Delta^{n}}
$$

is a Joyal fibration and DK-equivalence. In particular, it has the right-lifting-property with respect to $\varnothing \rightarrow \Delta^{0}$. This is equivalent to saying that any square

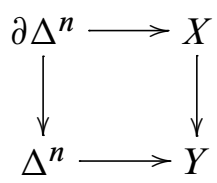

has a lifting. 


\section{Quillen equivalence of quasi-categories and simplicial cat- egories}

In this final section of the paper we use our previous results to establish the equivalence between the homotopy theories of quasi-categories and simplicial categories. This result was originally proven by Joyal [14] and Lurie [16].

8.1 Proposition For a map $X \rightarrow Y$ of simplicial sets, the following are equivalent:

(i) $f$ is a Joyal equivalence.

(ii) The map $\mathfrak{C}(f): \mathfrak{C}(X) \rightarrow \mathfrak{C}(Y)$ is a weak equivalence of simplicial categories.

(iii) $f$ is a $D K$-equivalence.

Proof The implication (i) $\Rightarrow$ (ii) is [8, Proposition 6.6].

The equivalence of (ii) and (iii) can be argued as follows. First, note that both conditions are invariant under Joyal equivalence; that is, if

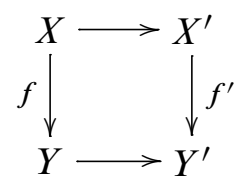

is a commutative square in which the horizontal maps are Joyal equivalences, then $f$ satisfies (ii) (resp. (iii)) if and only if $f^{\prime}$ does. For condition (iii) this is built into the definition, whereas for condition (ii) it follows from the fact (i) $\Rightarrow$ (ii). It is therefore enough to prove that (ii) and (iii) are equivalent under the assumption that $X$ and $Y$ are quasi-categories.

But recall from Proposition 2.20 that if $X$ is a quasi-category then $[*, X]_{E^{1}}$ is in bijective correspondence with the isomorphism classes in $\pi_{0} \mathfrak{C}(X)$. Also, we know by Corollary 5.3 that for any $a, b \in X$ the simplicial set $\operatorname{hMap}_{s \varphi_{e t, *}}\left(\Delta^{1}, X_{a, b}\right)$ is connected to $\mathfrak{C}(X)(a, b)$ by a natural zigzag of Kan equivalences. The equivalence of (ii) and (iii) now follows at once from the definitions.

Finally, we prove (ii) $\Rightarrow$ (i). Let $Y \stackrel{\sim}{\rightarrow} \hat{Y}$ be a fibrant-replacement in $s \mathscr{S e t}_{J}$, and then factor the composite $X \rightarrow Y \rightarrow \hat{Y}$ as a Joyal acyclic cofibration followed by a Joyal fibration. This produces a square

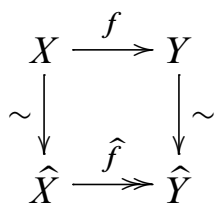


in which the vertical maps are Joyal equivalences (and therefore become weak equivalences after applying $\mathfrak{C}$ ). It follows that $\hat{f}$ also becomes a weak equivalence after applying $\mathfrak{C}$, and therefore $\hat{f}$ is a DK-equivalence by (ii) $\Rightarrow$ (iii). Then by Proposition $7.6 \hat{f}$ is a Joyal equivalence, and hence the same is true for the original map $f$ (by two-out-of-three applied to the above square).

8.2 Corollary The adjoint functors $\mathfrak{C}: \mathscr{C}_{e} t_{J} \rightleftarrows s^{\mathscr{C} a t}: N$ are a Quillen equivalence.

Proof The functor $\mathfrak{C}$ preserves cofibrations and acyclic cofibrations by $[8,6.4,6.6]$. So the functors $(\mathfrak{C}, N)$ are a Quillen pair. For the Quillen equivalence, the hard work has been done in the preceding pages and we now can follow the same argument as in [16]. We must prove two things, the first saying that for any fibrant simplicial category $\mathscr{D}$, the map

$$
\mathfrak{C}(N \mathscr{D}) \rightarrow \mathscr{D}
$$

is a weak equivalence in $s \mathscr{C} a t$. This has already been done in Proposition 5.9.

The second thing to be proven is that for any simplicial set $K$ and any fibrantreplacement $\mathfrak{C}(K) \rightarrow \mathscr{D}$ in $s \mathscr{C}$ at, the induced map

$$
K \rightarrow N \mathfrak{C}(K) \rightarrow N \mathscr{D}
$$

is a Joyal equivalence. By Proposition 8.1, it is enough to show instead that

$$
\mathfrak{C}(K) \rightarrow \mathfrak{C} N \mathfrak{C}(K) \rightarrow \mathfrak{C}(N \mathscr{D})
$$

is a weak equivalence in $s \mathfrak{b a t}$. But now we consider the larger diagram

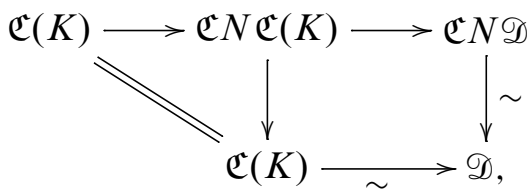

where the right vertical map is a weak equivalence by Proposition 5.9. It follows at once that the desired map is also a weak equivalence.

\section{Leftover proofs}

In this section we give two combinatorial proofs which were postponed in Section 4.

Recall from Section 1.3 that for a finite totally-ordered set $S=\left\{a_{0}, \ldots, a_{n}\right\}$ we let $\Delta^{S}$ denote the $n$-simplex whose ordered vertex set is $S$. Likewise $\Lambda_{a_{i}}^{S}$ denotes the horn of $\Delta^{S}$ consisting of all maximal faces containing $a_{i}$. Finally, it is convenient to also use the notation $\left[a_{0}, \ldots, a_{n}\right]$ to denote $\Delta^{S}$. 
9.1 Lemma For any necklace $T$, the maps $\operatorname{Spi}[T] \hookrightarrow T \hookrightarrow \Delta[T]$ are both Joyal equivalences.

Proof We will argue that the map $\Delta^{n} \vee \Delta^{1} \rightarrow \Delta^{n+1}$ is a composite of cobase changes along inner horn inclusions (ie, that the map is inner anodyne). By induction the same is therefore true for $\operatorname{Spi}\left[\Delta^{r}\right] \hookrightarrow \Delta^{r}$, for any $r>0$. The map $\operatorname{Spi}[T] \hookrightarrow \Delta[T]$ is precisely one of these maps, therefore it is a Joyal equivalence.

The fact that $\operatorname{Spi}[T] \rightarrow T$ is a Joyal equivalence can then be proven, bead by bead, using cobase changes of the maps $\operatorname{Spi}\left[\Delta^{r}\right] \rightarrow \Delta^{r}$. The desired result follows by two-out-of-three.

So everything follows once we have shown that $\Delta^{n} \vee \Delta^{1} \hookrightarrow \Delta^{n+1}$ is inner anodyne. Let $X=\Delta^{n+1}$ and define a filtration on $X$ by

$$
\begin{gathered}
X^{0}=[0,1, \ldots, n] \cup[n, n+1], \quad X^{1}=X^{0} \cup \bigcup_{i<n}[i, n, n+1], \\
X^{2}=X^{1} \cup \bigcup_{i<j<n}[i, j, n, n+1],
\end{gathered}
$$

and so on. That is, $X^{0}=\Delta^{n} \vee \Delta^{1}$ and $X^{i+1}$ is the union of $X^{i}$ and all $(i+2)-$ simplices of $X$ which contain $n$ and $n+1$. Note that $X^{n-1}=X$ and $X^{n-2}=\Lambda_{n}^{n+1}$. One readily checks that each inclusion $X^{i} \hookrightarrow X^{i+1}$ is a cobase change of $\left(\begin{array}{c}n \\ i+1\end{array}\right)$ inner horn inclusions. For instance, the inclusion $X^{0} \hookrightarrow X^{1}$ is obtained by gluing the 2-simplices $[i, n, n+1]$ to $X^{0}$ along their inner horns $\Lambda_{n}^{\{i, n, n+1\}}$, one 2-simplex for each $i \in\{0,1, \ldots, n-1\}$. The inclusion $X^{1} \hookrightarrow X^{2}$ is obtained by gluing the 3-simplices $[i, j, n, n+1]$ to $X^{1}$ along their inner horns $\Lambda_{n}^{\{i, j, n, n+1\}}$, and so forth.

The above proof is very typical of other proofs we will have to give, both later in this section and in the appendices. They involve starting with an inclusion $A \hookrightarrow B$ and producing a filtration

$$
A=B^{0} \subseteq B^{1} \subseteq \cdots \subseteq B^{m}=B
$$

in which every $B^{i} \hookrightarrow B^{i+1}$ is checked to be a cobase change of inner horn inclusions. This "checking" will usually be left to the reader, but we offer a few words of advice about this. Usually $B$ will be a simplicial set with the property that no two distinct simplices have the same ordered sequence of vertices. Examples of such include $\Delta^{n}$, $E^{1}$, as well as subcomplexes and products of such things. Note that this property will be inherited by all subcomplexes of $B$.

Suppose that $C \hookrightarrow B$ and $C^{\prime}$ is obtained from $C$ by pushing out $\Lambda_{k}^{n} \rightarrow \Delta^{n}$ along a map $f: \Lambda_{k}^{n} \rightarrow C$; we say that $C^{\prime}$ is obtained from $C$ by filling a horn. If the composite 
$\Lambda_{k}^{n} \rightarrow C \rightarrow B$ extends over $\Delta^{n}$, there will be an induced map $C^{\prime} \rightarrow B$. This will be an inclusion if and only if $f$ is a nonbounding horn in the sense of the following definition.

9.2 Definition A map $\Lambda_{k}^{n} \rightarrow C$ is called a nonbounding horn in $C$ if it does not extend to a map $\partial \Delta^{n} \rightarrow C$.

Faced with a given filtration $B^{0} \subseteq B^{1} \subseteq \cdots \subseteq B^{m}=B$, one typically checks that each $B^{i+1}$ is constructed from $B^{i}$ by pushing out along nonbounding inner horns. When no two distinct simplices of $B$ have the same ordered set of vertices, this can usually be done by inspection. The "nonbounding" condition serves as a verification that the $B^{i}$, s really do form a filtration as claimed.

Our next goal is to complete the proof of Proposition 4.5 by showing that for any $n \geq 1$ the canonical maps $C_{R}^{n} \rightarrow \Delta^{1}, C_{L}^{n} \rightarrow \Delta^{1}$, and $C_{\text {cyl }}^{n} \rightarrow \Delta^{1}$ are all Joyal equivalences. The proof will proceed by a combinatorial argument similar to what we just described.

Given integers $0 \leq k<n$, define $\Delta_{k}^{n}$ to be the quotient of $\Delta^{n}$ obtained by collapsing the initial $\Delta^{k}$ to a point and the terminal $\Delta^{n-k-1}$ to a (different) point. Note that $\Delta_{k}^{n}$ has exactly two vertices, and there is a unique surjection $\Delta_{k}^{n} \rightarrow \Delta^{1}$. Note also that $\Delta_{n-1}^{n}=C_{R}^{n}$ and $\Delta_{0}^{n}=C_{L}^{n}$.

9.3 Lemma For integers $0 \leq k<n$, the surjection $\Delta_{k}^{n} \rightarrow \Delta^{1}$ is a Joyal equivalence.

Proof Note that a nondegenerate simplex in $\Delta_{k}^{n}$ is either the first vertex, the last vertex, or is "uncollapsed" under the quotient $\Delta^{n} \rightarrow \Delta_{k}^{n}$. In this case it can be identified as the image of a unique nondegenerate simplex of $\Delta^{n}$ which we can denote by its sequence of vertices in $\Delta^{n}$. A vertex sequence $\left[v_{0}, \ldots, v_{p}\right]$ represents an uncollapsed simplex if and only if $v_{0} \leq k$ and $v_{p}>k$.

We first do the case $k=0$; let $X=\Delta_{0}^{n}$. Define a filtration on $X$ by

$$
X^{0}=[0,1], \quad X^{1}=\bigcup_{1<i \leq n}[0,1, i], \quad X^{2}=\bigcup_{1<i<j \leq n}[0,1, i, j],
$$

and so on. Note that $X^{n-1}=X$. It is easy to check that $X^{i} \hookrightarrow X^{i+1}$ is a cobase change along $\left(\begin{array}{c}n-1 \\ i+1\end{array}\right)$ inner horn inclusions $(0 \leq i \leq n-2)$, and therefore is a Joyal equivalence. So $X^{0} \hookrightarrow X$ is also a Joyal equivalence, and the desired result follows by two-out-of-three.

The proof in the case $k=n-1$ is completely symmetric to the $k=0$ case.

It remains to tackle the case $0<k<n-1$. Consider the inclusion $\Delta^{n-1} \hookrightarrow \Delta^{n}$ given by $[1,2, \ldots, n]$, and the induced inclusion $\Delta_{k-1}^{n-1} \hookrightarrow \Delta_{k}^{n}$. We may assume by 
induction that $\Delta_{k-1}^{n-1} \rightarrow \Delta^{1}$ is a Joyal equivalence, so it suffices to prove the same for $\Delta_{k-1}^{n-1} \hookrightarrow \Delta_{k}^{n}$. We will prove that this map is inner anodyne.

Define a filtration on $X=\Delta_{k}^{n}$ by $X^{0}=\Delta_{k-1}^{n-1}$ and

$$
\begin{gathered}
X^{1}=X^{0} \cup \bigcup_{k<j_{1}}\left[0,1, j_{1}\right], \quad X^{2}=X^{1} \cup \bigcup_{k<j_{1}<j_{2}}\left[0,1, j_{1}, j_{2}\right], \\
X^{3}=X^{2} \cup \bigcup_{k<j_{1}<j_{2}<j_{3}}\left[0,1, j_{1}, j_{2}, j_{3}\right],
\end{gathered}
$$

and so on. So $X^{i}$ is the union of $X^{i-1}$ and all $(i+1)$-simplices of $X$ containing 0 and 1. It is again easy to see that each $X^{i} \hookrightarrow X^{i+1}$ is a cobase change along nonbounding inner horn inclusions (the 1 -horn in each case), and so $X^{0} \hookrightarrow X^{n-1}=X$ is inner anodyne.

9.4 Proposition For every $n \geq 0$, the maps $C_{R}^{n} \rightarrow \Delta^{1}, C_{L}^{n} \rightarrow \Delta^{1}$, and $C_{\text {cyl }}^{n} \rightarrow \Delta^{1}$ are Joyal equivalences.

Proof The cases of $C_{R}^{n}$ and $C_{L}^{n}$ follow immediately from Lemma 9.3. For $C_{\text {cyl }}^{n}$ we argue as follows. Let $\{0,1, \ldots, n\}$ and $\left\{0^{\prime}, 1^{\prime}, \ldots, n^{\prime}\right\}$ denote the vertices in $\Delta^{n} \times\{0\}$ and $\Delta^{n} \times\{1\}$, respectively. Note that each simplex of $\Delta^{n} \times \Delta^{1}$ is completely determined by its vertices, and that $\Delta^{n} \times \Delta^{1}$ contains exactly $n+1$ nondegenerate $(n+1)$-simplices. For example if $n=2$ we have:

$$
\Delta^{2} \times \Delta^{1}=\Delta^{\left\{0,1,2,2^{\prime}\right\}} \cup \Delta^{\left\{0,1,1^{\prime}, 2^{\prime}\right\}} \cup \Delta^{\left\{0,0^{\prime}, 1^{\prime}, 2^{\prime}\right\}} .
$$

Let $D_{i}$ denote the $(n+1)$-simplex $\Delta^{\left\{0,1, \ldots, i, i^{\prime}, \ldots, n^{\prime}\right\}}$, for $0 \leq i \leq n$.

Recall that $C_{\text {cyl }}^{n}$ is obtained from $\Delta^{n} \times \Delta^{1}$ by collapsing any simplex whose vertices are contained in $\{0, \ldots, n\}$ or in $\left\{0^{\prime}, \ldots, n^{\prime}\right\}$. Let $E_{i}$ be the image of $D_{i}$ in $C_{\text {cyl }}^{n}$, and note that $E_{i}$ is isomorphic to $\Delta_{i}^{n+1}$.

Define a filtration on $X=C_{\text {cyl }}^{n}$ by setting

$$
X^{i}=E_{0} \cup E_{1} \cup \cdots \cup E_{i} .
$$

Note that $X^{n}=X$. We will prove by induction that the composite $X^{i} \hookrightarrow X \rightarrow \Delta^{1}$ is a Joyal equivalence, for every $i$. The base case $i=0$ is covered by Lemma 9.3. 
Note that $X^{i+1}=X^{i} \cup E_{i+1}$, and $E_{i+1} \cap X^{i} \cong \Delta_{i}^{\left\{0,1, \ldots, i,(i+1)^{\prime}, \ldots, n^{\prime}\right\}}$. This gives us a diagram in which both squares are pushouts:

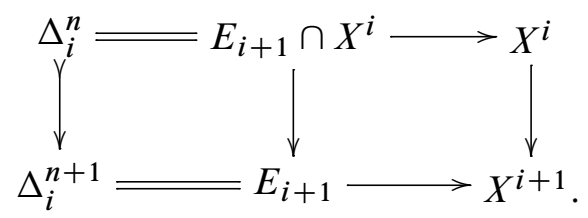

The left vertical map is a Joyal acyclic cofibration (using Lemma 9.3 and two-out-ofthree), so the same is true for $X^{i} \hookrightarrow X^{i+1}$. This completes the proof.

\section{Appendix A The box product lemmas}

In these appendices we develop all the properties of quasi-categories used in this paper, from first principles. This material is a summary of Joyal's work [15], and culminates in the proof of the existence of the Joyal model category structure (Theorem 2.13). Note that the only results in this paper which directly rely on the appendices occur in Section 2.

Appendix A is totally self-contained: we prove two box product lemmas, one yielding Proposition 2.6 and the other serving as a key step for Proposition 2.3(a). Appendix B is also self-contained: there we prove every unjustified result of Section 2 except for Theorem 2.13. Appendix $\mathrm{C}$ proves this final theorem. For the arguments in Appendix A and Appendix B, the reader may find it helpful to first read the discussion on nonbounding horns from Section 9.

A.1 Lemma The box product $\left(\Lambda_{k}^{n} \hookrightarrow \Delta^{n}\right) \square\left(\partial \Delta^{r} \hookrightarrow \Delta^{r}\right)$ is inner anodyne when $0<k<n$ and $0 \leq r$.

Proof Let $Y=\Delta^{n} \times \Delta^{r}$ and let $Y^{0}=\left(\Lambda_{k}^{n} \times \Delta^{r}\right) \amalg_{\Lambda_{k}^{n} \times \partial \Delta^{r}}\left(\Delta^{n} \times \partial \Delta^{r}\right)$. We will produce a filtration $Y^{0} \subseteq Y^{1} \subseteq \cdots \subseteq Y^{r+1}=Y$ and prove that each $Y^{i} \hookrightarrow Y^{i+1}$ is inner anodyne.

Let us establish some notation. An $m$-simplex $y$ in $Y$ is determined by its vertices, and we can denote it in the form

$$
y=\left[\begin{array}{llll}
a_{0} & a_{1} & \ldots & a_{m} \\
u_{0} & u_{1} & \ldots & u_{m}
\end{array}\right]
$$

where $0 \leq a_{i} \leq a_{i+1} \leq n$ and $0 \leq u_{i} \leq u_{i+1} \leq r$, for $0 \leq i<m$. Here we are writing $\left[\begin{array}{l}a \\ u\end{array}\right]$ instead of the usual $(a, u)$, because in the above notation the faces and degeneracies are obtained by just omitting or repeating columns. The simplex $y$ is degenerate if 
and only if two successive columns are identical. Note that two distinct nondegenerate simplices of $Y$ will not have any horn in common.

One checks that the simplex $y$ is an element of $Y^{0}$ if and only if it satisfies one of the following two conditions:

(i) $k \in\left\{a_{0}, a_{1}, \ldots, a_{m}\right\}$ and $\left\{a_{0}, a_{1}, \ldots, a_{m}\right\} \neq\{0,1, \ldots, n\}$.

(ii) $\left\{u_{0}, \ldots, u_{m}\right\} \neq\{0,1, \ldots, r\}$.

Let $Y^{1}$ be the union of $Y^{0}$ together with all simplices that contain the vertex $\left[\begin{array}{c}k \\ 0\end{array}\right]$, and in general let $Y^{i}$ be the union of $Y^{i-1}$ together with all simplices containing $\left[\begin{array}{c}k \\ i-1\end{array}\right]$. Note that $Y^{r+1}=Y$ : this follows from the fact that every simplex of $Y$ is a face of a simplex that contains some $\left[\begin{array}{l}k \\ i\end{array}\right]$.

Our goal is to show that each inclusion $Y^{i} \hookrightarrow Y^{i+1}$ is inner anodyne, and we will do this by producing another filtration

$$
Y^{i}=Y^{i}[n-1] \subseteq Y^{i}[n] \subseteq \cdots \subseteq Y^{i}[n+r]=Y^{i+1} .
$$

Notice that every simplex of $Y$ of dimension $n-1$ or less, containing $\left[\begin{array}{l}k \\ i\end{array}\right]$, lies in $Y^{0}$ (it satisfies condition (i)). For $t>n-1$ we define $Y^{i}[t]$ to be the union of $Y^{i}[t-1]$ and all nondegenerate simplices of $Y$ that have dimension $t$ and contain $\left[\begin{array}{l}k \\ i\end{array}\right]$. We claim that $Y^{i}[t] \hookrightarrow Y^{i}[t+1]$ is a cobase change of a disjoint union of inner horn inclusions; justifying this will conclude our proof.

Let $y$ be a nondegenerate simplex of $Y$ of dimension $t+1$, where $t \geq n-1$, and assume $y$ contains $\left[\begin{array}{c}k \\ i\end{array}\right]$ but $y \notin Y^{i}[t]$. Then every face of $y$ except possibly for the $\left[\begin{array}{l}k \\ i\end{array}\right]$-face is contained in $Y^{i}[t]$. We must show that $Y^{i}[t]$ cannot contain this final face of $y$, and also that this final face is not simultaneously filled by another inner horn in $Y^{i}[t]$; the latter is clear by the last sentence of the second paragraph of this proof, so we will concentrate on the former.

Write $y$ in the form of (A.2), and consider the column immediately preceding the $\left[\begin{array}{l}k \\ i\end{array}\right]$. This column cannot have $k$ in the top row, for then the bottom row would be at most $i-1$ and we would have $y \in Y^{i-1}$. Likewise, the top entry of this column cannot be $k-2$ or less or we would have $y \in Y^{0}$. So immediately preceding the $\left[\begin{array}{c}k \\ i\end{array}\right]$-column is a $\left[\begin{array}{c}k-1 \\ ?\end{array}\right]$-column. This cannot be a $\left[\begin{array}{c}k-1 \\ j\end{array}\right]$-column for $j<i$, since otherwise $y$ would be a face of the $(t+2)$-dimensional simplex obtained by inserting a $\left[\begin{array}{c}k \\ i-1\end{array}\right]$-column before the $\left[\begin{array}{c}k \\ i\end{array}\right]$; this would imply that $y \in Y^{i-1}$, giving a contradiction. So the $\left[\begin{array}{c}k \\ i\end{array}\right]$-column is preceded by $\left[\begin{array}{c}k-1 \\ i\end{array}\right]$.

Now consider the $\left[\begin{array}{l}k \\ i\end{array}\right]$-face of $y$; call this face $d y$. Note that the set of entries in the second row of $d y$ is the same as the corresponding set for the second row of $y$. A little 
thought then shows that $d y$ cannot satisfy condition (i) or (ii) above, since $y$ did not. So $d y \notin Y^{0}$. Then the only way $d y$ could be in $Y^{i-1}$ is if it were added somewhere along the way, but every simplex that was added was part of a simplex containing a $\left[\begin{array}{l}k \\ j\end{array}\right]$ for $j<i$; and clearly $d y$ is not part of any such simplex. So $d y \notin Y^{i-1}$. Finally, the $t$-dimensional simplices in $Y^{i}[t]-Y^{i-1}$ all contain $\left[\begin{array}{c}k \\ i\end{array}\right]$, and therefore $d y$ is not one of these either. Hence $d y \notin Y^{i}[t]$, and this shows that adjoining $y$ to $Y^{i}[t]$ amounts to filling a nonbounding horn, which is inner because $k \notin\{0, n\}$. This completes the argument.

The previous lemma allows us to wrap up a loose end from Section 2.

Proof of Proposition 2.6 This follows immediately from the preceding lemma, by a standard argument.

We next turn to box products of maps with $\{0\} \hookrightarrow E^{1}$. Recall that in Section 2 we introduced the notion of a quasi-isomorphism in a quasi-category $X$. It is convenient to extend this notion to all simplicial sets $S$ by saying that a 1 -simplex $e$ in $S$ is a quasi-isomorphism if for every quasi-category $X$ and every map $S \rightarrow X$, the image of $e$ in $X$ is a quasi-isomorphism as previously defined. Note that if $e: \Delta^{1} \rightarrow S$ extends to a map $E^{1} \rightarrow S$, then $e$ is necessarily a quasi-isomorphism.

A.3 Definition A map $\ell: \Lambda_{k}^{n} \rightarrow X$ is called a special right horn (resp. special left horn) if $k=n$ (resp. $k=0)$ and $\ell\left(\Delta^{\{n-1, n\}}\right)$ (resp. $\ell\left(\Delta^{\{0,1\}}\right)$ ) is a quasi-isomorphism in $X$. A special outer horn is defined to be either a special left horn or a special right horn. Finally $\ell$ is a special horn if it is either a special outer horn or any inner horn.

A map $f: X \rightarrow Y$ is called special outer anodyne if it is the composition of cobase extensions along special outer horns. The map $f$ is called special anodyne if it is the composition of cobase extensions along special horns.

The next box product lemma will be a key step in proving Proposition 2.3(a). Note the restriction of $r \geq 1$, which will be important later.

A.4 Lemma For any $r \geq 1$ the box product $f=\left(\{0\} \hookrightarrow E^{1}\right) \square\left(\partial \Delta^{r} \rightarrow \Delta^{r}\right)$ is special anodyne.

Proof Let $Y=E^{1} \times \Delta^{r}$ and let $Y^{0}=\left(\{0\} \times \Delta^{r}\right) \amalg_{\{0\} \times \partial \Delta^{r}}\left(E^{1} \times \partial \Delta^{r}\right)$. We will produce a filtration of simplicial sets $Y^{0} \subseteq Y^{1} \subseteq \cdots \subseteq Y^{r+1}=Y$ such that $Y^{i} \hookrightarrow Y^{i+1}$ is inner anodyne for $0 \leq i<r$ and special anodyne for $i=r$. 
Let us establish some notation. It is convenient to denote the 0 -simplices of $E^{1}$ by $a$ and $b$ rather than 0 and 1 . Note that every simplex of $E^{1}$ is uniquely determined by its vertices, and so the same is true of $Y$. The vertices of $Y$ are of the form $(a, i)$ or $(b, i)$ for $0 \leq i \leq r$; to ease the typography we will denote these $a_{i}$ and $b_{i}$, respectively. The vertex sequence of a simplex $y$ in $Y$ corresponds to a sequence of $a_{0}$ 's and $b_{0}$ 's, followed by a sequence of $a_{1}$ 's and $b_{1}$ 's, and so on up through the final sequence of $a_{r}$ 's and $b_{r}$ 's. We refer to the portion of the sequence consisting of the $a_{i}$ 's and $b_{i}$ 's as the " $i$-group"; note that this can be empty. Said differently, a simplex $y$ of $Y$ can be denoted by its vertex sequence

$$
y=\left(\left[y_{0}^{1}, \ldots, y_{0}^{k_{0}}\right],\left[y_{1}^{1}, \ldots, y_{1}^{k_{1}}\right], \ldots,\left[y_{r}^{1}, \ldots, y_{r}^{k_{r}}\right]\right)
$$

where for each $0 \leq i \leq r$ we have $0 \leq k_{i}$ and each $y_{i}^{j}$ is either the vertex $a_{i}$ or $b_{i}$. Note that the superscripts only serve as counters. Similarly, the brackets are not part of the data here; they are written for the ease of the reader. The " $i$-group" of $y$ corresponds to the sequence inside of the $i$-th set of brackets in (A.5). Note that the simplex $y$ is degenerate if and only if there exists $0 \leq i \leq r$ and $1 \leq j \leq k_{i}-1$ such that $y_{i}^{j}=y_{i}^{j+1}$-that is, if and only if there is a repetition inside one of the groups.

Let $Y^{m}$ denote the simplicial subset of $Y$ consisting of all simplices $y \in Y$ such that one of the following conditions is satisfied:

(i) There exists $0 \leq i \leq r$ such that $k_{i}=0$ (ie, some group is empty).

(ii) For all $i \geq m$ and all $1 \leq j \leq k_{i}$, one has $y_{i}^{j}=a_{i}$ (the $m$-group and higher consist only of $a$ 's).

The reader should check that $Y^{0}$ and $Y^{r+1}$ agree with our previous definitions. For $Y^{0}$, check that simplices in $\left(\{0\} \times \Delta^{r}\right)$ satisfy (ii) and simplices in $E^{1} \times \partial \Delta^{r}$ satisfy (i). For $Y^{r+1}=Y$, check that condition (ii) is vacuously satisfied for all simplices, when $m=r+1$.

Consider the inclusion $Y^{m} \hookrightarrow Y^{m+1}$ for some $0 \leq m \leq r-1$ (the case $m=r$ will be handled separately). The simplices in $Y^{m+1}-Y^{m}$ are of the form

$$
x=\left(\left[x_{0}^{1}, \ldots, x_{0}^{k_{0}}\right], \ldots,\left[x_{m}^{1}, \ldots, x_{m}^{k_{m}}\right],\left[a_{m+1}^{1}, \ldots, a_{m+1}^{k_{m+1}}\right], \ldots,\left[a_{r}^{1}, \ldots, a_{r}^{k_{r}}\right]\right)
$$

where $a_{i}^{j}=a_{i}$ and such that there is at least one $b$ in the $m$-group. Note that every such nondegenerate simplex is the face of a nondegenerate simplex of the form

$$
x^{\prime}=\left(\left[x_{0}^{1}, \ldots, x_{0}^{k_{0}}\right], \ldots,\left[x_{m}^{1}, \ldots, x_{m}^{k_{m}}, a_{m}\right],\left[a_{m+1}^{1}, \ldots, a_{m+1}^{k_{m+1}}\right], \ldots,\left[a_{r}^{1}, \ldots, a_{r}^{k_{r}}\right]\right) .
$$


Define an infinite sequence

$$
Y^{m}=Y^{m}[0] \subseteq Y^{m}[1] \subseteq Y^{m}[2] \subseteq \cdots
$$

whose union is $Y^{m+1}$ by letting $Y^{m}[t]$ be the union of $Y^{m}[t-1]$ and all $t$-simplices of the form $x^{\prime}$ above.

We claim that each $Y^{m}[t] \hookrightarrow Y^{m}[t+1]$ is a cobase change of a coproduct of inner horn inclusions. To prove this we'll show that every nondegenerate simplex $x^{\prime} \in$ $Y^{m}[t+1]-Y^{m}[t]$ comes from filling a unique nonbounding horn in $Y^{m}[t]$.

By the " $a_{m}$-face" of $x^{\prime}$ we mean the face obtained by removing the final $a_{m}$ in the $m$-th group. It is clear that every other face of $x^{\prime}$ lies in $Y^{m}[t]$, so we need only show that this $a_{m}$-face, which we denote $D$, does not lie in $Y^{m}[t]$. Since the $m$-group contains a $b_{m}$, we know that $D \notin Y^{m}$, and since it is a $t$-simplex it must have been added by filling a horn at stage $t$. This means that the $m$-group of $D$ is of the form $\left[x_{m}^{1}, \ldots, x_{m}^{k_{m}}, a_{m}\right]$ and hence the $m$-group of $x^{\prime}$ is of the form $\left[x_{m}^{1}, \ldots, x_{m}^{k_{m}}, a_{m}, a_{m}\right]$. But such a simplex is degenerate, contrary to assumption on $x^{\prime}$.

This completes our analysis of $Y^{m} \hookrightarrow Y^{m+1}$ for $0 \leq m<r$. When $m=r$ the same idea works, but the horns involved are special horns (they are special because every 1simplex within a single group - in particular, the $r$-th group —is a quasi-isomorphism). In fact one literally copies the previous paragraphs, replacing all instances of the word "inner" with the word "special." There is one subtlety that occurs, which is why one needs the hypothesis $r \geq 1$. In passing from $Y^{0}$ to $Y^{1}$, the first stage of the argument involves attaching the simplex $\left[a_{0}, a_{1}, \ldots,\left[b_{r}, a_{r}\right]\right]$ along its $a_{r}$-horn. But in the case $r=0$ this is a 1 -horn of a 1 -simplex, which is not allowed. This problem does not appear when $r \geq 1$, and so this completes the proof.

\section{Appendix B Special outer horns and applications}

Quasi-categories do not satisfy the lifting condition for general outer horns. But it turns out they do satisfy the lifting condition for special outer horns-outer horns where a particular map is a quasi-isomorphism. The purpose of this section is to prove various lifting results related to this phenomenon.

\section{B.1 The quasi-isomorphism lemmas}

Let $X$ be a quasi-category and let $f$ be a 1 -simplex in $X$. Recall that a 1 -simplex $h$ is a right inverse (or right quasi-inverse) for $f$ if there exists a $\sigma: \Delta^{2} \rightarrow X$ such that 
$d_{0}(\sigma)=f, d_{1}(\sigma)$ is degenerate, and $d_{2}(\sigma)=h$. We call the $2-$ simplex $\sigma$ a right inverse provider for $f$.

Similarly, $h$ is a left inverse for $f$ if there exists a $\tau: \Delta^{2} \rightarrow X$ with $d_{2}(\tau)=f, d_{1}(\tau)$ degenerate, and $d_{0}(\tau)=h$; and $\tau$ is called a left inverse provider for $f$.

In Proposition 2.18 we stated that if $X$ is a quasi-category and $f, g$, and $h$ are 1simplices in $X$, then $g f=h$ in $\pi_{0} \mathfrak{C}(X)$ if and only if there is a 2-simplex $m: \Delta^{2} \rightarrow X$ with $d_{0} m=g, d_{1} m=h$, and $d_{2} m=f$. Here is the proof:

Proof of Proposition 2.18 Let $X$ be a quasi-category. Define a relation on the $1-$ simplices of $X$ by $f \sim g$ if there exists a map $\sigma: \Delta^{2} \rightarrow X$ with $d_{1}(\sigma)=g$, $d_{2}(\sigma)=f$, and $d_{0}(\sigma)$ is degenerate. It is proven in [3, Lemma 4.11] (as well as [15]) that this gives an equivalence relation, and that there is a category $\operatorname{Ho}(X)$ where the maps are equivalence classes of 1 -simplices. It is then easy to produce maps of categories $\operatorname{Ho}(X) \rightarrow \pi_{0} \mathfrak{C}(X)$ and $\pi_{0} \mathfrak{C}(X) \rightarrow \operatorname{Ho}(X)$ showing that the two categories are isomorphic.

Now suppose that $f, g$, and $h$ are 1-simplices of $X$ and that $g f=h$ in $\pi_{0} \mathfrak{C}(X)$. Let $\sigma: \Delta^{2} \rightarrow X$ be any 2-simplex with $d_{0}(\sigma)=g$ and $d_{2}(\sigma)=f$ (such a simplex exists by the quasi-category condition). Let $u=d_{1}(\sigma)$. Then $u$ and $h$ will represent the same map in $\pi_{0} \mathfrak{C}(X)$, so we must have $u \sim h$. Thus, there is a $\tau: \Delta^{\{0,2,3\}} \rightarrow X$ with $d_{2}(\tau)=h, d_{3}(\tau)=u$, and $d_{0}(\tau)$ degenerate. We obtain in this way a map $F: \Lambda_{2}^{3} \rightarrow X$ which equals $\sigma$ on $[0,1,2], \tau$ on $[0,2,3]$, and is a degeneracy of $g$ on $[1,2,3]$. Extending $F$ to $\Delta^{3}$ and restricting to $[0,1,3]$ gives the desired 2-simplex with boundary $(g, h, f)$.

B.2 Proposition Let $X$ be a quasi-category, and let $f: \Delta^{1} \rightarrow X$. Then the following conditions are equivalent:

(i) $f$ is a quasi-isomorphism.

(ii) $f$ has a left inverse and a (possibly different) right inverse.

(iii) The image of $f$ in $\pi_{0} \mathfrak{C}(X)$ is an isomorphism.

Proof Clearly (i) $\Rightarrow$ (ii) $\Rightarrow$ (iii), and (iii) $\Rightarrow$ (i) is immediate by Proposition 2.18 .

\section{B.3 Special outer horn lifting}

Suppose given a map $f: \Lambda_{0}^{n} \rightarrow X$ such that $f([0,1])$ is a quasi-isomorphism. Since $f$ has an inverse, one could imagine producing a corresponding horn in which the 
direction of $f$ has been "flipped". This would be an inner horn, which could be extended to an $n$-simplex $\Delta^{n} \rightarrow X$. One could imagine flipping the $[0,1]$ simplex again to create an extension of the original map $f$.

Although vague, the above paragraph gives an idea of why quasi-categories should have liftings for special outer horns. Attempting to prove this result in the above manner results in a combinatorial nightmare. A different approach, using some clever techniques of Joyal [15], allows one to simplify it.

Given two maps $f: A \hookrightarrow B$ and $g$ : $C \hookrightarrow D$, let us use the notation $f \otimes g$ for the map

$$
(A \star D) \amalg_{A \star C}(B \star C) \hookrightarrow B \star D .
$$

B.4 Lemma Let $A \hookrightarrow B$ be a monomorphism of simplicial sets. Then for any $n \geq 0$ and any $0<k \leq n$, the map

$$
\left(\Lambda_{k}^{n} \hookrightarrow \Delta^{n}\right) \otimes(A \hookrightarrow B)
$$

is inner anodyne. (Note that the case $k=n$ is allowed.)

Proof By a routine argument one reduces to the case where $A \hookrightarrow B$ is $\partial \Delta^{r} \hookrightarrow \Delta^{r}$. So we are looking at the map

$$
\left(\Lambda_{k}^{n} \star \Delta^{r}\right) \amalg_{\Lambda_{k}^{n} \star \partial \Delta^{r}}\left(\Delta^{n} \star \partial \Delta^{r}\right) \hookrightarrow \Delta^{n} \star \Delta^{r} .
$$

Note that the codomain may be identified with $\Delta^{\{0,1, \ldots, n, n+1, \ldots, n+r+1\}}$. Under this identification $\Delta^{n} \star \partial \Delta^{r}$ is the union of the faces $[0,1, \ldots, n, n+1, \ldots, \hat{i}, \ldots, n+r+1]$ where $n+1 \leq i \leq n+r+1$. Likewise, the simplicial set $\Lambda_{k}^{n} \star \Delta^{r}$ is the union of the faces $[0,1, \ldots, \hat{i}, \ldots, n, n+1, \ldots, n+r+1]$ where $0 \leq i \leq n$ and $i \neq k$. It follows that the domain of the map in (B.5) is just $\Lambda_{k}^{\{0,1, \ldots, n+r+1\}}$, and so our map is an inner horn (even if $k=n$ or $r=0$ ).

Let $K$ be a simplicial set. As pointed out in [15, Section 5.2], the join functor $(-) \star K: s \mathscr{Y}_{e t} \rightarrow\left(K \downarrow s \mathscr{S}_{e t}\right)$ has a right adjoint. This right adjoint sends a simplicial set $X$ with a map $K \rightarrow X$ to a simplicial set denoted $X_{/ K}$; the $n$-simplices of $X_{/ K}$ are the maps $\Delta^{n} \star K \rightarrow X$ which extend the given map $K \rightarrow X$. Note that $X_{/ \varnothing}=X$. The following lemma will be useful:

B.6 Lemma Let $A \rightarrow X$ be a map of simplicial sets and let $X \rightarrow Y$ be an inner fibration. Then $X_{/ A} \rightarrow Y_{/ A}$ is also an inner fibration. In particular, if $X$ is a quasicategory then so is $X_{/ A}$.

Proof This is immediate from Lemma B.4, using adjointness. 
B.7 Proposition Let $X$ be a quasi-category and let $K \rightarrow X$ be a map of simplicial sets. Given $f: \Delta^{1} \rightarrow X_{/ K}$, let $f^{\prime}$ denote the composite $\Delta^{1} \rightarrow X_{/ K} \rightarrow X_{/ \varnothing}=X$. Then if $f^{\prime}$ is a quasi-isomorphism, so is $f$.

Proof We will prove that $f$ has a right inverse $g$. Then we will prove that $g$ itself has a right inverse. Therefore $g$ has a left and right inverse, hence is a quasi-isomorphism by Proposition B.2. Then $f$ is a quasi-isomorphism by two-out-of-three.

Choose a right inverse provider $\sigma: \Delta^{\{-1,0,1\}} \rightarrow X$ for $f^{\prime}: \Delta^{\{0,1\}} \rightarrow X:$ so $\left.\sigma\right|_{[0,1]}=f^{\prime}$ and $\left.\sigma\right|_{[-1,1]}$ is degenerate. Consider the lifting diagram

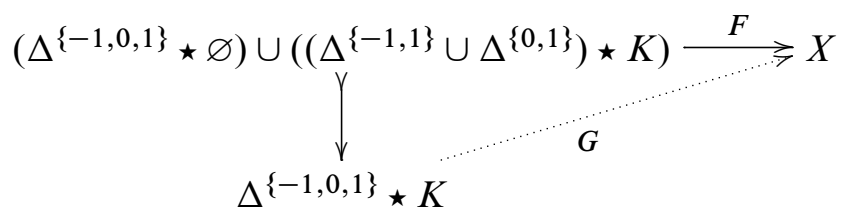

where the map $F$ is given by $\sigma$ on the left piece, the adjoint of $f$ on the $\Delta^{\{0,1\}} \star K$ piece, and on the remaining piece is equal to the composite

$$
\Delta^{\{-1,1\}} \star K \rightarrow \Delta^{\{1\}} \star K \hookrightarrow \Delta^{\{0,1\}} \star K \stackrel{f}{\longrightarrow} X .
$$

The vertical map in (B.8) is $\left(\Lambda_{1}^{\{-1,0,1\}} \hookrightarrow \Delta^{\{-1,0,1\}}\right) \otimes(\varnothing \hookrightarrow K)$, hence is inner anodyne by Lemma B.4. So there is a lifting $G$ in the above diagram. The adjoint $G^{\sharp}: \Delta^{\{-1,0,1\}} \rightarrow X_{/ K}$ of $G$ is a right inverse provider for $f$.

Let $g$ denote the map $\Delta^{\{-1,0\}} \rightarrow X_{/ K}$ obtained by restricting $G^{\sharp}$. So $g$ is a right inverse for $f$. The composite $g^{\prime}: \Delta^{\{-1,0\}} \rightarrow X_{/ K} \rightarrow X$ will be a right inverse for $f^{\prime}$. Hence $g^{\prime}$ is a quasi-isomorphism and itself has a right inverse. Repeating exactly the same argument as above, but replacing all occurrences of $f$ and $f^{\prime}$ by $g$ and $g^{\prime}$, we can construct a $k: \Delta^{1} \rightarrow X_{/ K}$ giving a right inverse for $g$. Returning to our outline from the first paragraph, this completes the proof.

The following is a restatement and proof of Proposition 2.4.

B.9 Proposition Let $X$ be a quasi-category and suppose given a solid arrow diagram

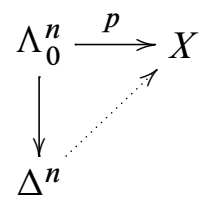

in which $p$ is a special left horn. Then there exists a dotted lift as shown.

A dotted lift also exists when $p$ is replaced by a special right horn $q: \Lambda_{n}^{n} \rightarrow X$. 
Proof We only prove the first statement; the second follows from a dual argument. To do so, we will produce a solid-arrow diagram

(B.10)

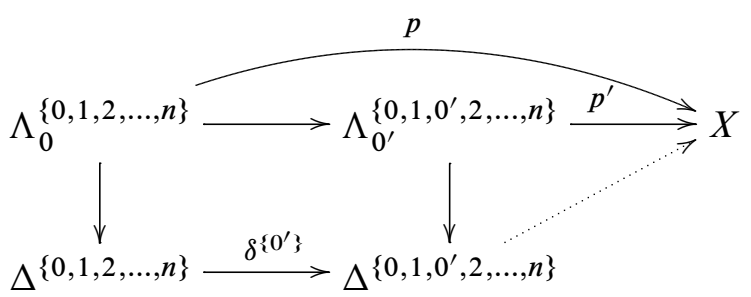

and the dotted arrow (which will exist because $X$ is a quasi-category) will compose with $\delta^{\left\{0^{\prime}\right\}}$ to produce the desired lift.

In order to construct $p^{\prime}$ we must extend $p: \Lambda_{0}^{n} \rightarrow X$ to $\Lambda_{0^{\prime}}^{\left\{0,1,0^{\prime}, 2, \ldots, n\right\}}$. Write $Z=\Lambda_{0^{\prime}}^{\left\{0,1,0^{\prime}, 2, \ldots, n\right\}}$ and $Z^{0}=\Lambda_{0}^{n}$. Let $Z^{1}=Z^{0} \cup\left[0,0^{\prime}, 2,3, \ldots, n\right]$, and let $Z^{2}=$ $Z^{1} \cup\left[\Delta^{\left\{0,1,0^{\prime}\right\}} \star \partial \Delta^{\{2,3, \ldots, n\}}\right]$. We now have a filtration

$$
Z^{0} \subseteq Z^{1} \subseteq Z^{2} \subseteq Z^{3}=Z,
$$

and we will construct $p^{\prime}$ on each term.

Extend $p$ to $Z^{1}$ via the composite $\Delta^{\left\{0,0^{\prime}, 2,3, \ldots, n\right\}} \rightarrow \Delta^{\{0,2,3, \ldots, n\}} \rightarrow X$ (in other words, via a degeneracy). Then consider the following diagram

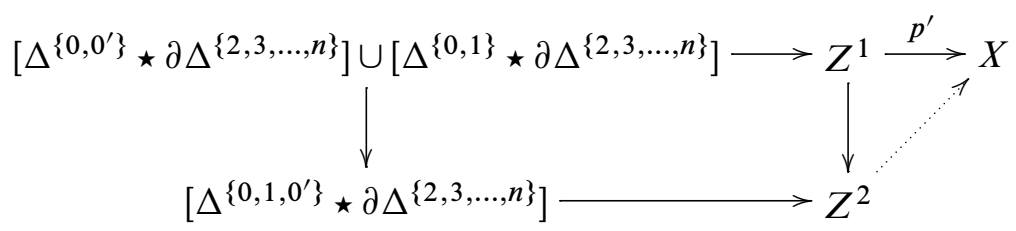

and note that the square is a pushout. By adjointness we need to construct a lift in the diagram

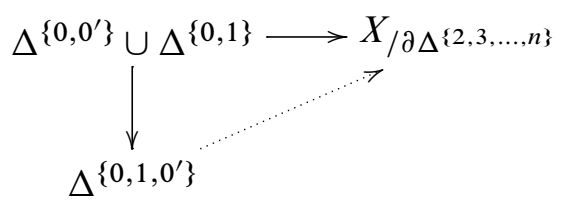

but such a lift exists by Proposition B.7 (applied to the quasi-isomorphism $\left.p\right|_{\Delta^{\{0,1\}}}$ ). After adjointing, this defines $p^{\prime}$ on $Z^{2}$.

Finally, we note that $Z^{2} \hookrightarrow Z^{3}$ is a cobase change of an inner horn inclusion. Indeed, the only nondegenerate simplex lying in $Z^{3}-Z^{2}$ is $\left[1,0^{\prime}, 2,3, \ldots, n\right]$, and all of its faces containing $0^{\prime}$ lie in $Z^{2}$. So $Z^{2} \hookrightarrow Z^{3}$ is a cobase change of $\Lambda_{0^{\prime}}^{\left\{1,0^{\prime}, 2, \ldots, n\right\}} \hookrightarrow$ $\Delta^{\left\{1,0^{\prime}, 2, \ldots, n\right\}}$. Since $X$ is a quasi-category, we may extend our lift $p^{\prime}$ from $Z^{2}$ to $Z^{3}$. This completes the construction of $p^{\prime}$, and thereby also completes the proof. 
We will also have need for a relative version of the above result:

B.11 Proposition Let $X$ and $Y$ be quasi-categories. Suppose given a solid arrow diagram

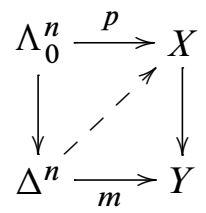

in which $p$ is a special left horn and $X \rightarrow Y$ is an inner fibration. Then there exists a dotted arrow making the diagram commute.

A dotted lift also exists when $p$ is replaced by a special right horn $q: \Lambda_{n}^{n} \rightarrow X$.

Before proving this we need a lemma, which takes care of a special case. The proof of the lemma uses Proposition B.9.

B.12 Lemma Let $X$ and $Y$ be quasi-categories, and suppose given a diagram

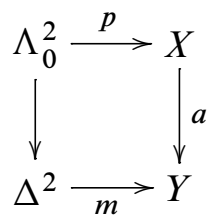

where $a$ is an inner fibration, $\left.p\right|_{[0,1]}$ is a quasi-isomorphism, and $\left.p\right|_{[0,2]}$ is degenerate. Then the above square has a lift.

A similar result holds when $p$ is a map $\Lambda_{2}^{2} \rightarrow X$ and $\left.p\right|_{[1,2]}$ is a quasi-isomorphism.

Proof We only prove the first statement, the second one being dual. Let $f=\left.p\right|_{[0,1]}$, and let $\sigma: \Delta^{\{0,1,2\}} \rightarrow X$ be a left inverse provider for $f$. Let $h=\left.\sigma\right|_{[1,2]}$.

Now define a map $\tau: \Lambda_{0}^{3} \rightarrow Y$ by $\left.\tau\right|_{[0,1,2]}=a \circ \sigma,\left.\tau\right|_{[0,1,3]}=m$, and where $\left.\tau\right|_{[0,2,3]}$ is a double degeneracy. Then since $\left.\tau\right|_{[0,1]}$ is a quasi-isomorphism (being the image of $f$ ), it follows by Proposition B.9 that we may extend $\tau$ to a map $\Delta^{3} \rightarrow Y$; call this extension $\tau$ as well.

As $X \rightarrow Y$ is an inner fibration, we may choose a lift in the following square:

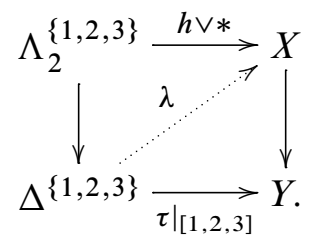


Extend $\lambda$ to a map $\Lambda_{2}^{\{0,1,2,3\}} \rightarrow X$ by $\left.\lambda\right|_{[0,1,2]}=\sigma$ and letting $\left.\lambda\right|_{[0,2,3]}$ be a double degeneracy. Then we have the diagram

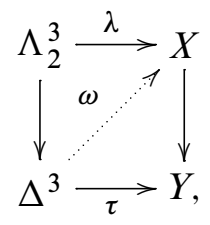

and there must exist a lifting $\omega$. One checks that $p=\left.\lambda\right|_{\Lambda_{0}^{\{0,1,3\}}}$ and $m=\left.\tau\right|_{\Delta^{\{0,1,3\}}}$, so $\left.\omega\right|_{[0,1,3]}$ provides the lift for our original square.

Proof of Proposition B.11 We only prove the first statement; the second follows from a dual argument. The proof follows the same general outline as that of Proposition B.9. We will produce a solid-arrow diagram

(B.13)

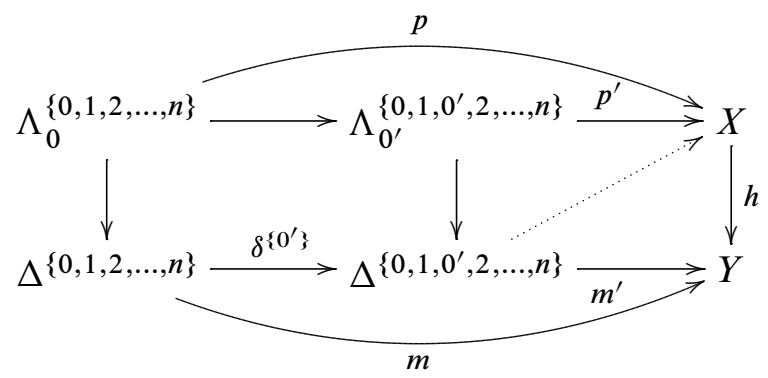

and the dotted arrow (which exists because $X \rightarrow Y$ is an inner fibration) will compose with $\delta^{\left\{0^{\prime}\right\}}$ to produce the desired lift.

Our map $m: \Delta^{n} \rightarrow Y$ may be regarded as a map $\Delta^{\{0,1\}} \star \Delta^{\{2,3, \ldots, n\}} \rightarrow Y$. The adjoint $f: \Delta^{\{0,1\}} \rightarrow Y_{/ \Delta^{\{2, \ldots, n\}}}$ is such that its composite with $Y_{/ \Delta^{\{2, \ldots, n\}} \rightarrow} \rightarrow Y$ is a quasi-isomorphism, hence by Proposition B.7 $f$ is itself a quasi-isomorphism. So there is a left inverse provider $\sigma: \Delta^{\left\{0,1,0^{\prime}\right\}} \rightarrow Y_{/ \Delta^{\{2, \ldots, n\}}}$ for $f$. Let $m^{\prime}$ be the adjoint of this map.

To construct $p^{\prime}$ we must produce a lift for the diagram

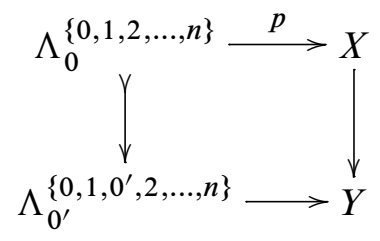

where the bottom horizontal map is the restriction of $m^{\prime}$. Define $Z^{0}, Z^{1}, Z^{2}$ and $Z^{3}$ exactly as in the proof of Proposition B.9; we will construct the lift $p^{\prime}$ on each $Z^{i}$. 
Extend $p$ to $Z^{1}$ via the composite $\Delta^{\left\{0,0^{\prime}, 2,3, \ldots, n\right\}} \rightarrow \Delta^{\{0,2,3, \ldots, n\}} \rightarrow X$. Continuing to argue as in the proof of Proposition B.9, extending $p^{\prime}$ to $Z^{2}$ amounts to constructing a lift in the diagram

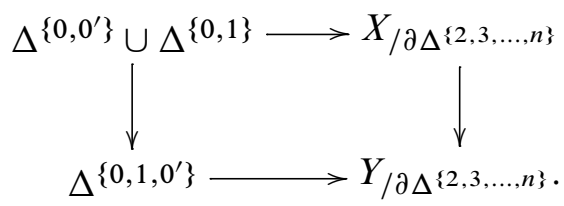

But this has a lift by Lemma B.12. After adjointing, this defines $p^{\prime}$ on $Z^{2}$.

Finally, the extension of $p^{\prime}$ from $Z^{2}$ to $Z^{3}$ follows exactly as in Proposition B.9. This completes the construction of $p^{\prime}$, and also completes the proof.

\section{B.14 Consequences of special outer horn lifting}

We have now shown that quasi-categories automatically have the right-lifting property for special outer horns. That is, we have established Proposition 2.4:

Proof of Proposition 2.4 This is precisely Proposition B.9.

In this subsection we prove a couple other consequences of outer-horn lifting that we will need elsewhere.

B.15 Proposition Let $f: X \rightarrow Y$ be an inner fibration between quasi-categories and suppose that $f$ has the $R L P$ with respect to $\{0\} \hookrightarrow E^{1}$. Then $f$ also has the RLP with respect to

$$
\left(\{0\} \hookrightarrow E^{1}\right) \square(A \hookrightarrow B)
$$

for any monomorphism $A \hookrightarrow B$. Equivalently, the map $X^{E^{1}} \rightarrow X^{\{0\}} \times_{Y^{\{0\}}} Y^{E^{1}}$ is a Kan acyclic fibration.

Proof By Lemma A.4 and Proposition B.11 we know that $f$ has the RLP with respect to $\left(\{0\} \hookrightarrow E^{1}\right) \square\left(\partial \Delta^{r} \hookrightarrow \Delta^{r}\right)$ for any $r>0$. The assumptions on $f$ take care of the case $r=0$. Since every monomorphism is generated by the boundary inclusions $\partial \Delta^{r} \hookrightarrow \Delta^{r}$, the result follows by an easy induction on simplices.

Proof of Proposition 2.3(a) This follows immediately from Proposition B.15, since $X \rightarrow *$ has the RLP with respect to $\{0\} \hookrightarrow E^{1}$.

Our last task is to prove Proposition 2.2. This is taken care of by the following lemma, which we learned from Nichols-Barrer [17]. 
B.16 Lemma Let $h: X \rightarrow Y$ be an inner fibration and suppose that $f: \Delta^{1} \rightarrow X$ is a quasi-isomorphism. Then for every solid-arrow commutative diagram of the form

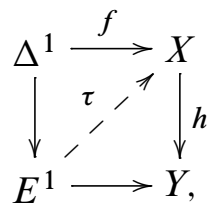

there exists a dotted arrow making the diagram commute.

Proof Each simplex in $E^{1}$ is determined by its sequence of vertices. We can therefore denote any $a \in E_{n}^{1}$ by a sequence $\left[a_{0}, a_{1}, \ldots, a_{n}\right]$ where each $a_{i} \in\{0,1\}$. Define a filtration $Z^{0} \subseteq Z^{1} \subseteq \cdots \subseteq E^{1}$ by letting $Z^{0}=[0], Z^{1}=[0,1], Z^{2}=[0,1,0]$, and so on. (Here we are identifying a simplex of $E^{1}$ with the simplicial subset of $E^{1}$ consisting of all faces and degeneracies of this simplex). The subset $Z^{n}$ is the unique nondegenerate $n$-simplex whose vertex sequence starts with 0 . Note that $E^{1}=\bigcup_{n \in \mathbb{N}} Z^{n}$.

We claim that for each $n \geq 1$ there is a pushout diagram

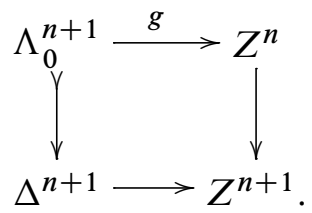

Here the map $g$ sends each simplex $[0,1, \ldots \hat{i} \ldots, n+1]$, for $0<i \leq n+1$, to the simplex in $E^{1}$ specified by reducing all the entries in the vertex sequence modulo 2 . It is easy to check that this reduction mod 2 is compatible on the overlap between simplices, therefore defines a map $\Lambda_{0}^{n+1} \rightarrow Z^{n}$, and that the pushout is $Z^{n+1}$.

Starting with a lifting square as in the statement of the lemma, one inductively produces lifts $Z^{n} \rightarrow X$ using special outer horn lifting (Proposition B.11). Taking the colimit gives the resulting lift $E^{1} \rightarrow Y$.

Proof of Proposition 2.2 We have already proved (ii) $\Longleftrightarrow$ (iii) as part of Proposition B.2; and (i) $\Rightarrow$ (ii) is obvious. Finally, (ii) $\Rightarrow$ (i) results immediately from applying Lemma B.16 to $X \rightarrow *$.

At this point we have proven all the assertions in Section 2 except for the existence of the Joyal model category structure. We take up that in the next section. 


\section{Appendix C Development of the Joyal model structure}

In this section we prove the existence of the Joyal model structure. The approach we follow is entirely due to Joyal [15], our only contribution being to streamline the presentation so that it occupies only a few pages.

Say that a map $X \rightarrow Y$ is a special inner fibration if it has the right-lifting-property with respect to all inner horn inclusions as well as with respect to the map $\{0\} \hookrightarrow E^{1}$. Note that such a map also has the RLP with respect to $\{1\} \hookrightarrow E^{1}$, using the unique nonidentity automorphism of $E^{1}$. Also note that if $X$ is a quasi-category then $X \rightarrow *$ is a special inner fibration (using the retraction $E^{1} \rightarrow\{0\}$ ).

C.1 Lemma Let $X$ and $Y$ be quasi-categories. If $X \rightarrow Y$ is a special inner fibration and $A \rightarrow B$ is a monomorphism, then $X^{B} \rightarrow X^{A} \times_{Y^{A}} Y^{B}$ is also a special inner fibration.

Proof From Proposition 2.6 and the Cartesian adjunction, $X^{B} \rightarrow X^{A} \times_{Y^{A}} Y^{B}$ is an inner fibration. Using Proposition B.15 we also know it has the RLP with respect to $\{0\} \hookrightarrow E^{1}$.

C.2 Lemma Let $f: X \rightarrow Y$ be a special inner fibration between quasi-categories, and assume that $f$ is also a Joyal equivalence. Then $f$ has the RLP with respect to both $\varnothing \rightarrow \Delta^{0}$ and $\{0,1\} \hookrightarrow E^{1}$.

Proof For a quasi-category $X$, let $J(X) \subseteq X$ be the subcomplex consisting of those simplices having the property that every 1 -dimensional face is a quasi-isomorphism. If Kan $\subseteq s$ Set denotes the full subcategory of Kan complexes, then $J: s \mathscr{Y} t \rightarrow$ Kan and this is the right adjoint of the inclusion functor Kan $\hookrightarrow s$ Set. In particular, note that $J$ preserves limits. The quasi-category $J(X)$ is the " $\infty$-groupoid of $\infty$-isomorphisms in $X$." Note as well that $X$ and $J(X)$ have the same 0 -simplices.

It is easy to see that since $X \rightarrow Y$ is a special inner fibration, $J(X) \rightarrow J(Y)$ is a Kan fibration (lifting with respect to inner horns is easy, and for outer horns follows from Proposition B.11). Moreover, $X \rightarrow Y$ is an $E^{1}$-homotopy equivalence by Remark 2.10. Since $J\left(X \times E^{1}\right) \cong J(X) \times J\left(E^{1}\right)=J(X) \times E^{1}$, it follows that $J(X) \rightarrow J(Y)$ is an $E^{1}$-homotopy equivalence as well. This implies that it is a Kan equivalence, therefore it is a Kan acyclic fibration. 
But now observe that any lifting square

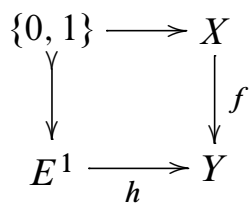

necessarily factors through $J(X) \rightarrow J(Y)$, and therefore has a lift. The same is true for lifting squares with $\varnothing \rightarrow \Delta^{0}$. This completes the proof.

We need one more lemma:

C.3 Lemma Let $X$ and $Y$ be quasi-categories.

(a) If $X \rightarrow Y$ is a special inner fibration and a Joyal equivalence, then it is a Kan acyclic fibration.

(b) If $A \rightarrow B$ is a monomorphism and a Joyal equivalence, then $X^{B} \rightarrow X^{A}$ is a Kan acyclic fibration.

Proof For (a), we will show that $X \rightarrow Y$ has the RLP with respect to any monomorphism $A \hookrightarrow B$. This is equivalent to proving that $X^{B} \rightarrow\left[X^{A} \times_{Y^{A}} Y^{B}\right]$ is surjective on 0 -simplices. Consider the diagram

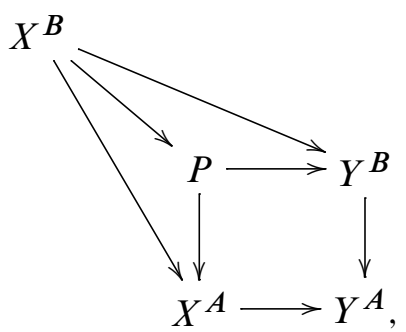

where $P$ is the pullback, and suppose $w$ is a $0-$ simplex of $P$. Write $w_{1}, w_{2}$, and $w_{3}$ for the images of $w$ in $Y^{B}, Y^{A}$, and $X^{A}$, respectively.

By Remark 2.10, $X \rightarrow Y$ is an $E^{1}$-homotopy equivalence. The functor $(-)^{B}$ is readily seen to preserve $E^{1}$-homotopy equivalences: one uses the natural maps $Z^{B} \times E^{1} \rightarrow$ $\left(Z \times E^{1}\right)^{B}$ adjoint to the composite ev $\times$ id: $B \times Z^{B} \times E^{1} \rightarrow Z \times E^{1}$. It follows that $X^{B} \rightarrow Y^{B}$ is also an $E^{1}$-homotopy equivalence. Hence $X^{B} \rightarrow Y^{B}$ is a Joyal equivalence, and it is a special inner fibration by Lemma C.1. By Lemma C.2 it is therefore surjective, so there is a 0 -simplex $x \in X^{B}$ whose image is $w_{1}$. Let $x_{3}$ 
denote the image of $x$ in $X^{A}$. Then $x_{3}$ and $w_{3}$ have the same image in $Y^{A}$, so we have a square

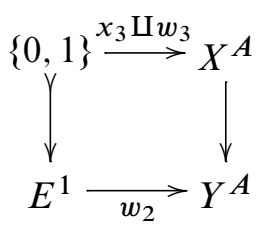

where the bottom map collapses everything to $w_{2}$. But $X^{A} \rightarrow Y^{A}$ is a special inner fibration and a Joyal equivalence (by the same arguments used for $X^{B} \rightarrow Y^{B}$ ), so Lemma C. 2 shows that the above square has a lifting $\lambda: E^{1} \rightarrow X^{A}$. Consider the induced map $\tilde{\lambda}: E^{1} \rightarrow P$ which projects to $w_{1}$ in $Y^{B}, w_{2}$ in $Y^{A}$, and $\lambda$ in $X^{A}$.

At this point we have a new square

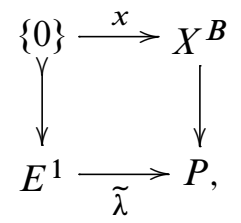

which has a lift because Lemma C.1 tells us that $X^{B} \rightarrow P$ is a special inner fibration. The image of the vertex 1 is the desired preimage of the original 0 -simplex $w$.

To prove (b), first note that $X^{B} \rightarrow X^{A}$ is a special inner fibration between quasicategories. Using (a), it suffices to show that this map is also a Joyal equivalence. Let $S$ be any simplicial set. Then $\left[B, X^{S}\right]_{E^{1}} \rightarrow\left[A, X^{S}\right]_{E^{1}}$ is a bijection for every quasi-category $X$. But $\left[B, X^{S}\right]_{E^{1}}=[B \times S, X]_{E_{1}}=\left[S, X^{B}\right]_{E^{1}}$, and similarly with $B$ replaced by $A$. Hence

$$
\left[S, X^{B}\right]_{E^{1}} \rightarrow\left[S, X^{A}\right]_{E^{1}}
$$

is a bijection for every simplicial set $S$. Taking $S=X^{A}$ and $S=X^{B}$ one sees at once that $X^{B} \rightarrow X^{A}$ is an $E^{1}$-homotopy equivalence, hence it is a Joyal equivalence by Remark 2.10 .

We now prove the existence of the Joyal model structure:

Proof of Theorem 2.13 We use a result of Jeff Smith's about producing model structures on locally presentable categories, written up by Beke. By [1, Theorem 1.7 and Proposition 1.15] we are guaranteed the existence of a cofibrantly-generated model structure on $s \mathscr{S}$ et where the cofibrations are the monomorphisms and the weak equivalences are the Joyal equivalences if we can verify the following: 
(1) The Joyal equivalences are closed under retracts and satisfy the two-out-of-three property.

(2) Every Kan acyclic fibration is a Joyal equivalence.

(3) The class of cofibrations which are Joyal equivalences is closed under pushouts and transfinite composition.

(4) The class of Joyal equivalences is an accessible class of maps, in the sense of [1, Definition 1.14].

The notion of accessible class of maps, needed in (4), depends on the category-theoretic notion of accessible category. The latter is a very technical condition that we will not recall here. All we need below is that the surjections in $\mathscr{S e t}$ form an accessible class of maps, and that if $F: \mathscr{C} \rightarrow \mathscr{D}$ is a functor between nice enough categories and $F$ preserves sufficiently large filtered colimits, then the inverse image under $F$ of an accessible class of maps is again accessible. The interested reader may consult Beke [1] for full details.

We now explain why conditions (1)-(4) are satisfied. For (2), if $f: X \rightarrow Y$ is a Kan acyclic fibration then there is a map $\chi: Y \rightarrow X$ such that $f \chi=\mathrm{id}$. Then the two maps $f \chi f$ and $f$ are equal, hence they are $E^{1}$-homotopic (trivially). So we have a square

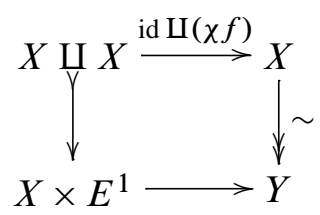

and this square must have a lifting. This shows that $f$ is an $E^{1}$-homotopy equivalence, hence it is a Joyal equivalence by Remark 2.10 .

We prove (3) by showing that a monomorphism $f: A \hookrightarrow B$ is a Joyal equivalence if and only if it has the left-lifting-property with respect to special inner fibrations between quasi-categories. Since monomorphisms and maps with a left-lifting-property are closed under pushouts and transfinite compositions, this will be enough.

First suppose that $f$ is a monomorphism with the indicated left-lifting-property. Then, in particular, it has this property with respect to the maps $X \rightarrow *$ and $X^{E^{1}} \rightarrow X^{\{0,1\}}$ for every quasi-category $X$ (using Lemma C.1). It follows immediately that $f$ is a Joyal equivalence. 
Now suppose that $f$ is a monomorphism and a Joyal equivalence. For any special inner fibration $X \rightarrow Y$ between quasi-categories, consider the diagram

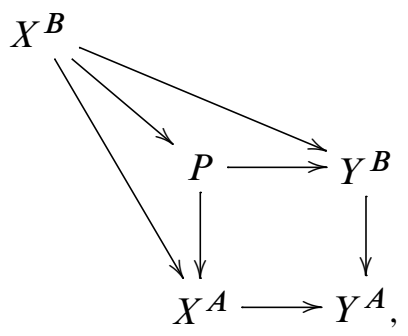

where $P=X^{A} \times_{Y^{A}} Y^{B}$. The map $X^{B} \rightarrow P$ is a special inner fibration by Lemma C.1. By Lemma C.3(b), the maps $Y^{B} \rightarrow Y^{A}$ and $X^{B} \rightarrow X^{A}$ are Kan acyclic fibrations. Hence the pullback $P \rightarrow X^{A}$ is also a Kan acyclic fibration. But then $P \rightarrow X^{A}$ and $X^{B} \rightarrow X^{A}$ are both Joyal equivalences, hence so is $X^{B} \rightarrow P$. By Lemma C.3(a) we have that $X^{B} \rightarrow P$ is an acyclic Kan fibration, hence it is surjective. But this precisely says that $A \rightarrow B$ has the left-lifting-property with respect to $X \rightarrow Y$. This now completes the proof of (3).

Finally, for point (4) we argue as follows. Let $S$ be the set consisting of all inner horn inclusions together with the map $\{0\} \hookrightarrow E^{1}$. Consider the functorial factorizations

$$
[X \stackrel{f}{\longrightarrow} Y] \mapsto\left[X \stackrel{i_{f}}{\longrightarrow} P_{f} \stackrel{p_{f}}{\longrightarrow} Y\right]
$$

provided by the small object argument. Here $X \rightarrow P_{f}$ is a transfinite composition of pushouts of coproducts of the maps in $S$, and $P_{f} \rightarrow Y$ has the right-lifting-property with respect to maps in $S$. It is an observation of Smith's (and a straightforward exercise) that these factorization functors preserve $\lambda$-filtered colimits for large enough regular cardinals $\lambda$. Also note that $X \rightarrow P_{f}$ is a Joyal equivalence, using point (3) together with the fact that the maps in $S$ are Joyal equivalences and cofibrations.

For brevity write $X \rightarrow L_{S}(X)$ for the factorization applied to $X \rightarrow *$. For $f: X \rightarrow Y$, let $R(f)$ be the map indicated in the following diagram:

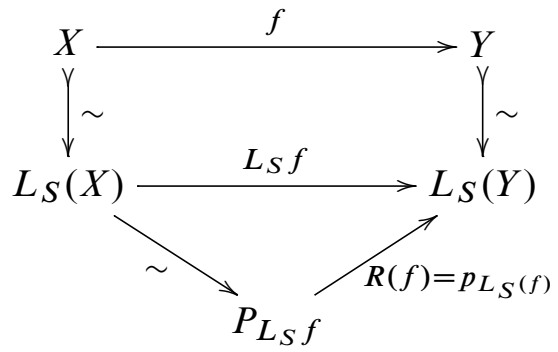


Note that $R(f)$ is a special fibration between quasi-categories, and that the indicated maps are Joyal equivalences. It is easy to see that $f$ is a Joyal equivalence if and only if $R(f)$ is a Joyal equivalence; by Lemma C.3(a) together with point (2), the latter is true if and only if $R(f)$ is a Kan acyclic fibration. So the class of Joyal equivalences is $R^{-1}(\mathscr{T})$, where $\mathscr{T}$ is the class of Kan acyclic fibrations. But $R$ is an accessible functor because it preserves large enough filtered colimits, and $\mathcal{T}$ is an accessible class by Lemma C.4 below. So by [1, Proposition 1.18] the class of Joyal equivalences is also accessible.

This completes the proof of the existence of a model structure where the cofibrations are the monomorphisms and the weak equivalences are the Joyal equivalences. To characterize the fibrant objects, note first that it follows at once from Proposition 2.11(c) that every fibrant object will be a quasi-category. Let $X$ be a quasi-category, and let $X \succ \hat{X}$ be a fibrant-replacement in our model structure. By Proposition 2.11(b), there is a lifting:

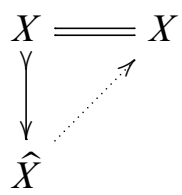

But then $X$ is a retract of the fibrant object $\hat{X}$, hence $X$ is fibrant.

For uniqueness of the model structure, see the explanation in [8, Section 2.3].

The following lemma was used in the above proof:

C.4 Lemma The class of Kan acyclic fibrations in sYet is an accessible class of maps in the sense of [1, Definition 1.14].

Proof It is easy to check that the category of surjections in $\mathscr{Y}$ et is an accessible class. Consider the functor $G: \operatorname{Mor}(s \mathscr{Y} e t) \rightarrow \operatorname{Mor}(\mathscr{Y} e t)$ which sends a map $f: X \rightarrow Y$ to the map

$$
\coprod_{n \geq 0}\left[X^{\Delta^{n}} \rightarrow X^{\partial \Delta^{n}} \times_{Y^{\partial \Delta^{n}}} Y^{\Delta^{n}}\right]
$$

restricted to the 0 -simplices. Note that $G$ preserves filtered colimits, because the functors $(-)^{\Delta^{n}}$ and $(-)^{\partial \Delta^{n}}$ do, and hence $G$ is an accessible functor. The class of Kan acyclic fibrations is exactly the inverse image under $G$ of the surjections; hence by [1, Proposition 1.18] the class of Kan acyclic fibrations is an accessible class of maps. 


\section{References}

[1] T Beke, Sheafifiable homotopy model categories, Math. Proc. Cambridge Philos. Soc. 129 (2000) 447-475 MR1780498

[2] J E Bergner, A model category structure on the category of simplicial categories, Trans. Amer. Math. Soc. 359 (2007) 2043-2058 MR2276611

[3] J M Boardman, R M Vogt, Homotopy invariant algebraic structures on topological spaces, Lecture Notes in Math. 347, Springer, Berlin (1973) MR0420609

[4] A K Bousfield, D M Kan, Homotopy limits, completions and localizations, Lecture Notes in Math. 304, Springer, Berlin (1972) MR0365573

[5] J-M Cordier, T Porter, Homotopy coherent category theory, Trans. Amer. Math. Soc. 349 (1997) 1-54 MR1376543

[6] D Dugger, Classification spaces of maps in model categories arXiv:math/0604537

[7] D Dugger, A primer on homotopy colimits, Preprint (2008) Available at http:// math. uoregon. edu/ ddugger/hocolim.pdf

[8] D Dugger, D I Spivak, Rigidification of quasi-categories, Alg. Geom. Topol. 11 (2011) 225-261

[9] W G Dwyer, D M Kan, Calculating simplicial localizations, J. Pure Appl. Algebra 18 (1980) 17-35 MR578563

[10] W G Dwyer, D M Kan, Function complexes in homotopical algebra, Topology 19 (1980) 427-440 MR584566

[11] W G Dwyer, D M Kan, Simplicial localizations of categories, J. Pure Appl. Algebra 17 (1980) 267-284 MR579087

[12] PS Hirschhorn, Model categories and their localizations, Math. Surveys and Monogr. 99, Amer. Math. Soc. (2003) MR1944041

[13] A Joyal, Quasi-categories and Kan complexes, J. Pure Appl. Algebra 175 (2002) 207222 MR1935979 Special volume celebrating the 70-th birthday of Professor Max Kelly

[14] A Joyal, Simplicial categories vs. quasi-categories, Preprint (2008)

[15] A Joyal, The theory of quasi-categories, Preprint (2008)

[16] J Lurie, Higher topos theory, Annals of Math. Studies 170, Princeton Univ. Press (2009) MR2522659

[17] J P Nichols-Barrer, On quasi-categories as a foundation for higher algebraic stacks, PhD thesis, Massachusetts Institute of Technology (2007) Available at http:// proquest . umi. com/pqdlink?FMT=7\&DID=1372017801\&RQT=309 
Department of Mathematics, University of Oregon Eugene OR 97403, USA

Department of Mathematics, Massachusetts Institute of Technology

Building 2, Room 236, 77 Massachusetts Avenue, Cambridge MA 02139-4307, USA

ddugger@uoregon.edu, dspivak@math.mit.edu

http://pages.uoregon.edu/ddugger/, http://math.mit.edu/ dspivak/

Received: 22 December 2009 Revised: 16 August 2010 